\title{
Use of Multiscale Zirconium Alloy Deformation Models in Nuclear Fuel Behavior Analysis
}

\author{
Robert Montgomery ${ }^{\mathrm{a}, *}$, Carlos Toméb, Wenfeng Liuc, Alankar Alankar ${ }^{\mathrm{d}}$, Gopinath Subramanian ${ }^{\mathrm{e}}$, \\ Christopher Stanek ${ }^{\mathrm{b}}$ \\ ${ }^{a}$ Pacific Northwest National Laboratory \\ ${ }^{b}$ Los Alamos National Laboratory \\ ${ }^{c}$ ANATECH Corporation \\ ${ }^{d}$ Indian Institute of Technology Bombay \\ ${ }^{e}$ University of Southern Mississippi
}

\begin{abstract}
Accurate prediction of cladding mechanical behavior is a key aspect of modeling nuclear fuel behavior, especially for conditions of pellet-cladding interaction (PCI), reactivity-initiated accidents (RIA), and loss of coolant accidents (LOCA). Current approaches to fuel performance modeling rely on empirical constitutive models for cladding creep, growth and plastic deformation, which are limited to the materials and conditions for which the models were developed. To improve upon this approach, a microstructurally-based zirconium alloy mechanical deformation analysis capability is being developed within the United States Department of Energy Consortium for Advanced Simulation of Light Water Reactors (CASL). Specifically, the viscoplastic self-consistent (VPSC) polycrystal plasticity modeling approach, developed by Lebensohn and Tomé [1], has been coupled with the BISON engineering scale fuel performance code to represent the mechanistic material processes controlling the deformation behavior of light water reactor (LWR) cladding. A critical component of VPSC is the representation of the crystallographic nature (defect and dislocation movement) and orientation of the grains within the matrix material and the ability to account for the role of texture on deformation. A future goal is for VPSC to obtain information on reaction rate kinetics from atomistic calculations to inform the defect and dislocation behavior models described in VPSC. The multiscale modeling of cladding deformation mechanisms allowed by VPSC far exceed the functionality of typical semi-empirical constitutive models employed in nuclear fuel behavior codes to model irradiation growth and creep, thermal creep, or plasticity. This paper describes the implementation of an interface between VPSC and BISON and provides initial results utilizing the coupled functionality.
\end{abstract}

\section{Introduction}

The US DOE's Consortium for Advanced Simulation of Light Water Reactors (CASL) program is developing multiphysics, multi-dimensional modeling and analysis capabilities to assess safety margins and the impact of plant operation on nuclear fuel rod behavior. These activities include an enhanced version of the engineering scale fuel behavior code, BISON, that is being developed to model the thermal, mechanical, and chemical behavior of LWR fuel rods during normal operation, operational transients, and hypothetical accidents [2]. Integral to this endeavor is the research and development activities to expand mechanistic material behavior modeling that builds on the recent advances in computational material science, such as

\footnotetext{
${ }^{*}$ Corresponding Author

Email addresses: robert.montgomery@pnnl.gov (Robert Montgomery), tome@lanl.gov (Carlos Tomé), wenfeng.lui@anatech.com (Wenfeng Liu), alankar.alankar@iitb.ac.in (Alankar Alankar), gopinath.subramanian@usm.edu (Gopinath Subramanian), stanek@lanl.gov (Christopher Stanek)
} 
ab-initio methods using density functional theory to determine certain diffusion/interaction rate constants or microstructurally-aware crystal plasticity methods to represent dislocation behavior within individual grains. Significant improvements are possible in fuel performance modeling and analysis provided that expanded microscale models of the underlying physics controlling material behavior can be implemented.

This paper summarizes recent material modeling efforts to incorporate a microstructurally-based zirconium alloy mechanical deformation analysis capability into the BISON engineering scale fuel performance code. The viscoplastic self-consistent (VPSC) polycrystal plasticity modeling approach, developed by Lebensohn and Tomé [1], has the ability to represent the mechanistic material processes controlling the deformation behavior of the cladding, such as dislocation glide and climb, and dislocation-dislocation interactions. Furthermore, VPSC considers the crystallographic orientation of the grains within the matrix material that leads to anisotropic mechanical behavior of the cladding. These capabilities far exceed the functionality of typical semi-empirical constitutive models employed in fuel behavior codes to model irradiation growth and creep, thermal creep, or plasticity. While the empirical models for zirconium alloy creep, growth, and plasticity have been used over the years with large data sets for validation, limitations remain when changes are made to the material fabrication process, alloy composition, or the operational demand envelop to regimes of higher fast fluence, elevated temperatures, or increased applied stress. The use of a Zr-alloy constitutive model that is based on a mechanistic understanding of the microstructural characteristics can allow for extension of fuel performance modeling to applications of improved cladding materials or new operating regimes where validation data is limited.

The structure of the paper is as follows. Section 1 provides an overview of the current methods used to model cladding deformation during irradiation in engineering scale fuel performance codes and a description of the vision to incorporate an advance cladding deformation model. Section 2 summarizes the microstructually-aware constitutive model to represent the anisotropic deformation behavior of hexagonalclosed packed materials such as zirconium alloy cladding tubes. Section 3 describes the approach used to interface the advanced cladding deformation model with the structural mechanics module within a fuel rod behavior analysis capability. Section 4 summarizes the results from two different demonstration cases; irradiation growth and a high temperature thermal creep case. These demonstration cases highlight the advantages and features of using a mechanistic material constitutive model for cladding deformation behavior. Finally, Section 5 summarizes the accomplishments to date and provides a road map to developing a more complete implementation of a microstructurally-aware, atomistically-informed zirconium alloy cladding constitutive model for irradiation and stress induced deformations during normal operation and transient events in a LWR.

\subsection{Background}

The processes that control the behavior of fuel and cladding materials during irradiation consist of a complex interaction between pre-existing material microstructural features and the changes caused by the imposed thermal, mechanical, chemical, and irradiation conditions. Traditional fuel behavior modeling capabilities are built on a suite of empirical or semi-empirical material property or behavior models [3]. In most cases, the empirical models consider that the mechanisms of irradiation creep, irradiation growth, and thermal creep are uncoupled and contribute independently to the overall response of the cladding. These models have been derived from a variety of testing and characterization programs that contained limited sets of environmental conditions or material characteristics. While fuel and cladding material behavior under irradiation conditions are among the most complex physics to simulate and understand, there are many areas where improved mechanistic modeling can have significant impact in the realm of fuel behavior simulation and analysis. These areas include the diffusion of fission gas atoms in $\mathrm{UO}_{2}$ ceramic material, evolution of $\mathrm{UO}_{2}$ thermal conductivity under irradiation, and zirconium alloy deformation mechanisms resulting from fast neutron damage. This paper will focus on the advanced modeling of zirconium alloy cladding deformation.

The fuel cladding is the first barrier in the reactor to prevent the release of radioactive fission products and actinides into the environment. A typical light-water reactor contains approximately 50,000 to 80,000 cylindrical fuel rods in the reactor core. Each rod has a diameter of $\sim 10 \mathrm{~mm}(0.4 \mathrm{in})$ and are arranged in a square lattice called a fuel assembly to allow for coolant flow between the rods. A fuel assembly is composed of $\sim 100$ to 280 fuel rods with a length of $\sim 3800 \mathrm{~mm}(\sim 150$ inches) and the structural components used to 
maintain the assembly geometry and to allow insertion of the reactivity control system. The major parts of a LWR fuel rod are the zirconium alloy cladding tube ( $\sim 10 \mathrm{~mm}$ outer diameter and $\sim 0.6 \mathrm{~mm}$ wall thickness) and the roughly $300 \mathrm{UO}_{2}$ pellets that are vertically stacked in the cladding. Residence time within the reactor results in atomic scale changes in the materials of the fuel rod arising from interactions with the neutron radiation field, the fission products, and the chemical environments. Ultimately, sufficient modification of the materials will occur, leading to conditions of component failure that limit the lifetime performance of the fuel rod/assembly. Accounting for the evolution of the thermal, mechanical, and chemical properties of the zirconium-alloy cladding for LWR fuels is critical in demonstrating the safe and economic operation of nuclear reactors. Specifically, understanding the mechanical strength change of zirconium-alloys for inreactor conditions and the deformation behavior of zirconium-alloy cladding tubes under normal operation and accident conditions are of great value to the improvement of fuel performance and to the prevention of fuel rod failures.

Zirconium alloys exhibit anisotropic mechanical behavior that is a function of the fabrication process due to the hexagonal-closed packed (HCP) crystallographic structure of the material [4]. The thermomechanical processing variables result in a crystallographic texture that is important, both for throughput in the fabrication process and for the in-reactor mechanical behavior. The zirconium-alloy tube texture impacts the irradiation growth, thermal and irradiation creep, yield strength, and the anisotropy of plastic deformation. It is therefore important to capture the role of as-fabricated texture and irradiation damage on microstructural changes in the material constitutive models used to calculate the performance of fuel cladding during normal operation and accident conditions.

The primary areas of concern for understanding and predicting the in-reactor deformation behavior of LWR zirconium-alloy cladding are: irradiation growth, irradiation and thermal creep, and plastic deformation $[5,6]$. Irradiation growth primarily refers to the cladding tube elongation in the axial direction caused by the accumulation of irradiation damage. Even though irradiation growth can cause small changes (a few millimeters in the full length fuel rod at the end of life), the differential growth may lead to fuel rod bowing, rod to rod or rod to nozzle contact, and the potential for fuel rod failure.

The in-reactor creep and growth of zirconium-alloy cladding is a time-dependent deformation process under low stress conditions. Creep deformation is composed of two components, thermal creep and irradiationinduced creep $[7,8]$. These processes are complex functions of material microstructure and composition, temperature, irradiation damage, and stress. Irradiation complicates the creep processes by creating sessile dislocation loops that promote an athermal growth via climbing and by producing defects that retard the thermal creep through interaction between these defects and dislocations. The creep down of the cladding tube under the high coolant pressure at the beginning of life is primarily driven by irradiation-induced creep. Under increased stress conditions, creep strains can relax the stress applied on the cladding tube arising from pellet-clad mechanical contact. Stress relaxation is an important component of the processes that reduce the cladding failure potential during power maneuvers.

In postulated accident conditions, the mechanical deformation of the cladding can be large due to either high temperature or high stress[9]. The non-recoverable deformation caused by either creep or instantaneous plasticity becomes important for accident conditions because these deformations can affect the core coolable geometry or lead to ductile rupture or brittle fracture of the cladding.

\subsection{Current Empirical Modeling for Cladding Deformation}

Current nuclear fuel performance analysis codes model the deformation of the cladding tube as a mechanical equilibrium problem using either a thin-shell theory or using the finite element method[50, 11, 12]. Both approaches require a constitutive law to describe the material response under different types of deformation mechanisms, including thermal expansion, elastic deformation, thermal and irradiation-induced creep, irradiation growth, and instantaneous plasticity. Most constitutive models assume that these mechanical deformation processes are uncoupled and so additive. Under input variables of temperature, irradiation dose (fast neutron flux), and stress, a set of empirical equations are used to describe the contribution of the different deformation mechanisms to the overall dimensional changes of the cladding. 
Typically, the cladding strain rate during irradiation is represented as a summation of the various deformation mechanisms;

$$
\dot{\epsilon}_{t o t a l}=\dot{\epsilon}_{e l}+\dot{\epsilon}_{t h e x p}+\dot{\epsilon}_{g r}+\dot{\epsilon}_{c r e e p}+\dot{\epsilon}_{p l}
$$

where;

$\dot{\epsilon}_{e l}$ is the strain rate due to elastic deformation,

$\dot{\epsilon}_{\text {thexp }}$ is the strain rate due to thermal expansion,

$\dot{\epsilon}_{g r}$ is the strain rate due to irradiation growth,

$\dot{\epsilon}_{\text {creep }}$ is the strain rate due to thermal and irradiation creep, and

$\dot{\epsilon}_{p l}$ is the strain rate due to instantaneous plasticity.

As mentioned previously, each of these strain components are calculated using semi-empirical material models derived from experimental data obtained under a variety of conditions. Generally, separate effectsbased testing has been used to determine the deformation rates for irradiation-induced and thermal creep and irradiation growth mechanisms. Although, these tests are designed to produce stress-free conditions as a means to study the role of neutron damage on dimensional changes for irradiation growth, existing methods are unable to prevent the intragranular creep induced by evolving internal stresses.

The thermal creep data from zirconium alloys has been traditionally been fit using a Dorn-based expression [8] that relates the steady-state creep rate to the applied stress using a hyperbolic sine function raised to an exponent $n$ that ranges between 2 and 4 , depending on the stress level. The value of $n$ obtained from thermal creep tests suggests that the creep mechanisms transition from a self diffusion controlled process at low stresses (ie. Nabarro-Herring creep) to that of dislocation climb and glide at stresses greater than $\sim 0.5 \sigma_{\text {yield }}[13,14]$. The dependence on temperature is represented by an activation energy related to diffusion of defects. A comprehensive review of zirconium alloy creep has been prepared by Franklin, et al.[14]. Equation 2 below is used in several zirconium alloy cladding tube constitutive models employed in fuel behavior analyses to compute the steady state thermal creep rate during irradiation $[8,15]$;

$$
\dot{\epsilon}_{\text {creep }}^{s s}=A \frac{E}{T}\left(\sinh \frac{a_{i} \sigma_{e f f}}{E}\right)^{n} \exp \left(-\frac{Q}{R T}\right)
$$

where;

$\dot{\epsilon}_{\text {creep }}^{s s}$ is the steady state thermal creep strain rate

$E$ is the elastic modulus

$\sigma_{\text {eff }}$ is the effective stress

$T$ is the temperature

$R$ is the universal gas constant

$Q$ is the activation energy

$A$ and $a_{i}$ are material constants that depend on fabrication technique, alloy composition, and irradiation damage

The complex microstructure and texture of zirconium alloys has led to a variety of observations regarding the dependence of thermal creep rate on material chemistry, alloying elements, and fabrication processes. Important alloying elements affecting zirconium thermal creep, include tin, niobium, and oxygen. Also, experiments have shown that zirconium alloys exhibit time dependent creep, both primary and secondary thermal creep rates[13]. Typically, Equation 2 is modified using a time dependent strain hardening rule to 
account for primary thermal creep based on the approach developed by Amin, et al[16]. Total thermal creep strain, including the primary component is given by;

$$
\epsilon_{\text {total }}^{\text {th }}=\epsilon_{p}^{s}\left[1-\exp \left(K_{1}\left(\dot{\epsilon}_{\text {creep }}^{s s} \times t\right)^{K_{2}}\right)\right]+\dot{\epsilon}_{\text {creep }}^{s s} \times t
$$

where;

$\epsilon_{\text {creep }}^{t h}$ is the total thermal creep strain

$\epsilon_{p}^{s}$ is the saturated primary creep strain

$t$ is time

$K_{1}$ and $K_{2}$ are material constants derived from experimental data

Equations 2 and 3 provide a description of the thermal creep behavior of zirconium alloys and numerous researchers have applied these equations to experimental data to obtain the material constants. Irradiation damage has been shown to decrease the thermal creep strain rate and several have developed modifications to the material constants used in Equation 3 to include the effect of fast neutron damage[11, 15].

Irradiation-induced creep by largely athermal mechanisms is typically represented as a function proportional to the applied stress and fast neutron flux. Constants are used to accommodate material dependencies such as residual cold work and alloying elements[11, 15]. The irradiation-induced creep model used in engineering fuel behavior calculations is given by;

$$
\dot{\epsilon}_{c r e e p}^{i r r}=C_{\circ} \phi^{C_{1}} \sigma^{C_{2}}
$$

where;

$\dot{\epsilon}_{c r e e p}^{i r r}$ is the irradiation-induced creep rate

$\phi$ is the fast neutron flux

$\sigma$ is the applied stress

$C_{\circ}, C_{1}$ and $C_{2}$ are material constants derived from experiments.

Both $C_{1}$ and $C_{2}$ are near unity for most zirconium alloy cladding materials used today. Experiments have shown that the irradiation-induced creep rate is insensitive to temperature in the range of normal reactor operating conditions.

The total creep rate of the cladding is then given by the summation of Equation 3 (in differential form) and Equation 4;

$$
\dot{\epsilon}_{\text {creep }}=\dot{\epsilon}_{\text {creep }}^{t h}+\dot{\epsilon}_{\text {creep }}^{\text {irr }}
$$

Finally, stress free changes of geometry with constant volume have been observed in zirconium alloys under irradiation conditions. This condition, called irradiation growth, arises as a consequence of fast neutron damage forming networks of interstitial and vacancy dislocation loops that promote expansion in the prismatic direction of the zirconium HCP crystal and shrinkage in the basal direction $[5,7]$. At the engineering scale, irradiation growth manifests itself as cladding tube elongation, with a small contraction in the through thickness and hoop directions. The cladding tube elongation behavior is controlled by adjusting the microstructure of the material to have specific texture and dislocation density (cold work). Researchers have established that the growth strain of a polycrystalline material is strongly influenced by the orientation of the basal poles of the HCP crystal. As a consequence, the empirical models used for cladding tube elongation in fuel behavior codes are material type and fabrication route dependent.

The form of the cladding tube elongation models used to calculate the irradiation growth behavior of zirconium alloy tubes is given by;

$$
\epsilon_{g r}=D_{\circ}(\phi t)^{D_{1}}
$$

where 


\author{
$\epsilon_{g r}$ is the irradiation growth creep strain \\ $\phi$ is the fast neutron flux \\ $t$ is the time
}

$\phi t$ represents that fast neutron fluence

$D_{\circ}$ and $D_{1}$ are material constants that depend on the material composition and texture.

Data obtained from irradiated cladding tubes with and without fuel pellets has found that $D_{1}$ ranges between 0.5 for annealed material to 0.9 for cold-worked stress relieved material[6, 17]. A dependence of $D_{\circ}$ on both temperature and texture has been reported by several researchers. However, within the range of operating temperatures, the irradiation growth behavior appears to be insensitive to temperature. Experimental data at higher neutron fast fluence exposure have found that annealed zirconium alloys exhibit a break away or accelerated irradiation growth behavior. The increase in growth rate is associated with the formation of c-loop dislocations on the basal planes[18, 19]. Holt and others have developed microstructure based rate theory models that account for vacancy and interstitial point defect diffusion and interaction with dislocations to form $\langle a\rangle$ and $\langle c\rangle$ component loops [20, 21]. Most empirical growth rate models ignore this effect.

The empirical models outlined in Equations 1 through 6 above provide estimates of the cladding deformation during irradiation that is compatible with most of the computational requirements of fuel behavior modeling tools used to design fuel rod systems and operating conditions that ensure the safe and reliable performance of light water reactors $[10,22]$. However, the empirical constants that represent material dependencies used throughout these models require extensive observation and testing campaigns to obtain the necessary data to derive the values, limiting the applicability of the models to the range of cladding tube microstructure, temperature, irradiation dose and stress state conditions utilized for performing such fitting. Because of the need to gather this data on irradiated materials, the data collection leads to complicated and costly testing methods and post-irradiation material characterization. The difficulties of obtaining the necessary data to build these models has restricted the evolution of both the constitutive models used in fuel rod modeling codes and the engineering codes themselves. Furthermore, introduction of new materials is slowed by the need to perform extensive irradiation and testing programs to generate data for empirical model development. As a consequence, there is a need to improve our predictive capabilities for cladding deformation, and connect the basis for these models to physical material mechanisms and microstructure evolution during irradiation.

The recent advent of computational material science capabilities now provides the potential to improve or replace the empirical models used in calculating zirconium alloy cladding behavior during normal operation and accident conditions[23]. By addressing the more basic mechanistic processes that lead to material dimensional changes, such as interactions between dislocation networks and point defects, improvements in the constitutive models may allow for accommodating changes in material texture, cold working, and alloy composition without the need to generate costly experimental data.

\title{
1.3. CASL Advanced Modeling Approach
}

The fuel behavior model development activities on-going within the CASL program consists of three different length and time scales[24]: (1) the engineering scale 2D/3D finite element fuel behavior code to capture integral fuel rod behavior, (2) microscopic length scale model development to account for the texture, microstructure, alloy contents, and lattice damage network on cladding creep and growth, clad hydride formation, and waterside corrosion, and (3) atomistic length scale modeling to provide improved understanding of diffusion coefficients and chemical reaction kinetics associated with fission gas release, fission product attack of the cladding, and corrosion. A key focus of the current material modeling effort is to develop more in-depth physics-based models for irradiation creep and growth, thermal creep, outer cladding surface corrosion, inner surface cladding corrosion, fission product release and transport, and ceramic material fracture behavior. 
The BISON code was selected to perform the engineering scale fuel rod modeling. The BISON fuel performance code is an engineering scale finite element method (FEM) code that is built upon the MOOSE computational finite element framework [25, 26]. BISON uses both 2-D and 3-D geometric fuel rod representations and a material property and fuel behavior model library for the $\mathrm{UO}_{2}$ and Zircaloy system to accurately calculate the thermal, mechanical, and chemical processes active throughout a single LWR fuel rod during operation in a reactor. The fuel rod modeling efforts in the CASL program are being developed to evaluate the potential of cladding failure by several different phenomenological processes that occur during the lifetime operation of the fuel. All of these processes happen as a result of multi-physics and multi-scale interactions arising from the material response to the imposed temperature, stress, and radiation fields.

Several activities are underway within the CASL program to address the needed capabilities for advanced modeling of fuel behavior during normal, operational transients, and accident events. One of these efforts is the expansion of a microstructure-based deformation model using the Visco-Plastic Self-Consistent (VPSC) approach to calculate thermal and irradiation creep, irradiation growth, and plastic deformation of the cladding. A schematic of the microstructure-aware approach to cladding deformation modeling is shown in Figure 1. The approach consists of deploying an enhanced version of VPSC where the constitutive framework is based on dislocation density behavior (from the seminal work of Beyerlein and Tomé [27]). This capability is interfaced with the engineering scale fuel performance code, BISON to obtain the environmental conditions, macroscopic stress field, temperature, and neutron fluence. Finally, a future goal is for VPSC to obtain information on reaction rate kinetics from atomistic calculations. The approach outlined in Figure 1 represents a quantum leap forward in modeling the effects of the irradiation environment on cladding deformations.

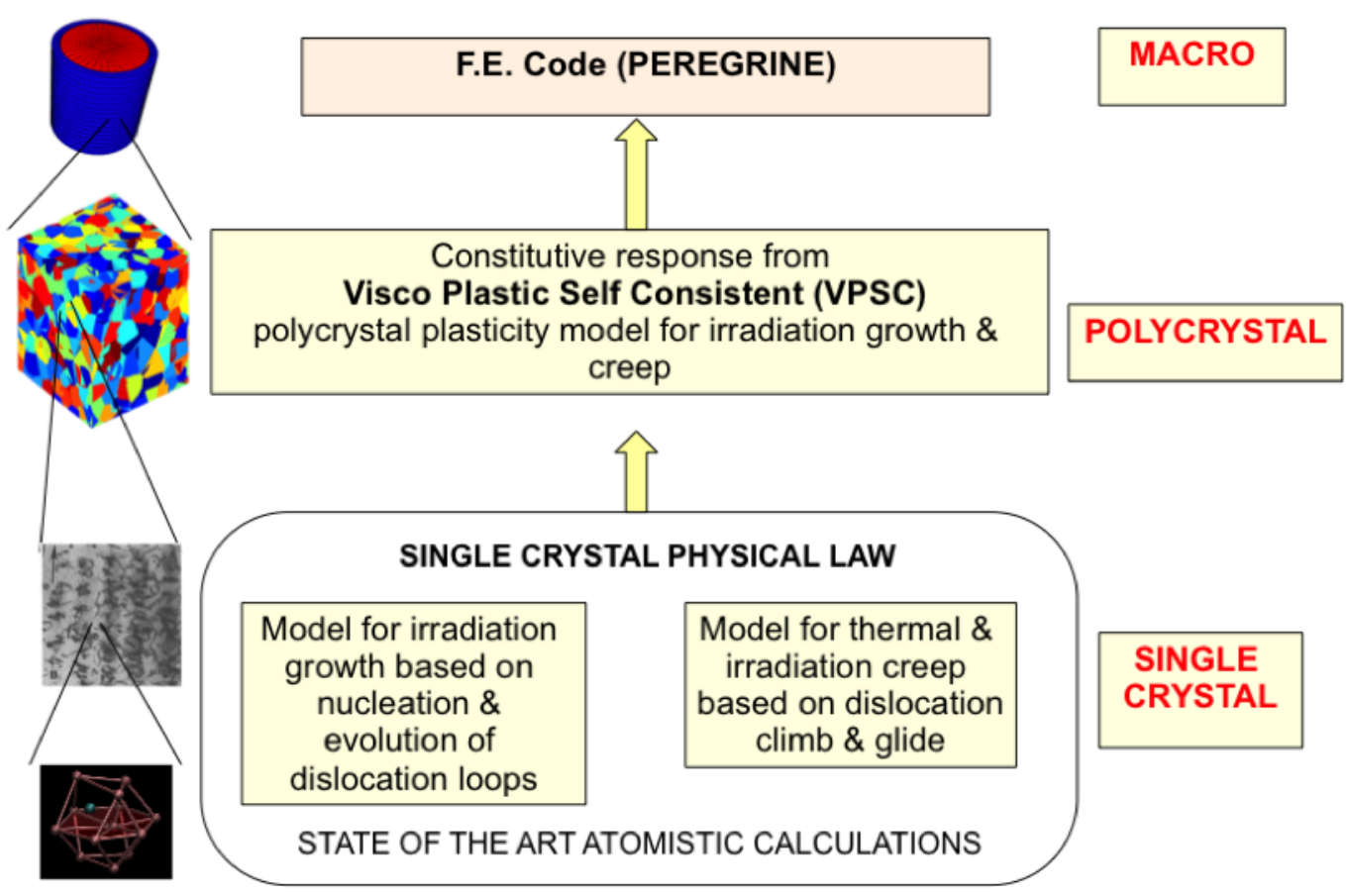

Figure 1: A schematic of the microstructurally-aware cladding deformation model approach under development by the authors

First, the single crystal formulation in VPSC accounts for the vacancy and interstitial loops in the cladding created by irradiation. It also accounts for the climb of those loops through defect capture, and for their interaction with mobile dislocations on active slip systems at the microscale. Second, the polycrystal model in VPSC then aggregates the effect of the active slip systems within single grains, and combining this with crystallographic texture information, calculates the dimensional changes in a representative volume of 
material. The constitutive equations in VPSC that account for stress or irradiation-induced deformation are functions of activation energies for dislocation motion or reaction constants (sink or emission strengths), respectively. The latter define the interaction kinetics between dislocations and other microstructure features such as interstitial atoms, vacancies, and grain boundaries.

While established values are available for the different interaction constants/parameters used in VPSC, the microstructure model framework provides a method to utilize information obtained from atomistic calculations that can expand or improve the formulations. Recently, Subramanian et al. [28] performed a series of atomistic simulations using both molecular dynamics and kinetic Monte Carlo methods to study the absorption rate of self interstitial atoms and vacancies by various sinks, such as edge dislocations within zirconium crystals. With further work, it is possible that this method will allow more accurate computation of kinetic parameters, such as sink strength and sink efficiency of dislocation networks that are used in traditional radiation damage models. Accurate computations of point defect absorption rates are critical for calculating mesoscale phenomena, such as dislocation climb and loop growth, which determine microstructural evolution under irradiation.

\section{Visco-Plastic Self Consistent Model Overview}

This section summarizes the models developed to describe the constitutive response of zirconium alloy material subjected to irradiation. The processes controlling zirconium alloy deformation can be separated into different regimes, depending on temperature and stress, involving the following mechanisms: 1) irradiation growth and irradiation creep dominate at reactor operating temperatures $\left(T<0.25 T_{M}\right)$ and low stresses $\left(\sigma<0.7 \sigma_{F}\right)$, and are mediated by the climb of dislocations (growth) and by climb-controlled-glide of dislocations (creep), and driven by a large concentration of irradiation-produced vacancies and interstitial atoms; 2) thermal creep $\left(T>0.3 T_{M}\right.$ or $\left.\sigma>0.7 \sigma_{F}\right)$, controlled by climb and glide of dislocations due to absorption/emission of vacancies, which are abundant at high temperature; and 3) instantaneous plasticity $\left(\sigma>0.9 \sigma_{F}\right)$ : dictated by generation and glide of dislocations with stress-induced activation over barriers. A rough deformation map would look like the one in Figure 2, with the understanding that the domains represent conditions where a particular mechanism prevails, but does not exclude other mechanisms from being active simultaneously.

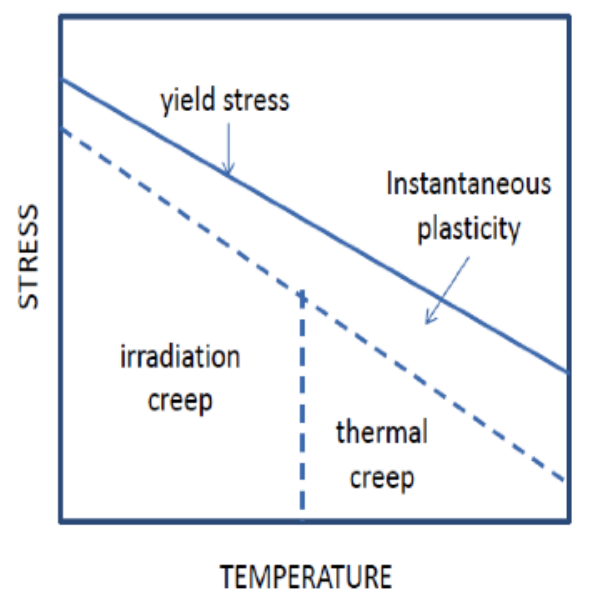

Figure 2: Schematic domains of prevailing mechanism in irradiated Zr alloys as a function of temperature and stress

Because all the deformation mechanisms are mediated by dislocations, and because dislocations exhibit specific orientations in the Zr crystals, a Crystal Plasticity (CP) model of the material has been adopted, which accounts not only for the directionality of dislocations in the crystal lattice, but also for the distribution 
of crystal orientations in the cladding tube (texture). In addition to explicitly accounting for anisotropy, such a CP model has the advantage of being based on the actual physical mechanisms of dislocation glide and climb (creep and instantaneous plasticity), dislocation-dislocation interaction (hardening), dislocationvacancy interaction, and dislocation interstitial interaction (growth).

\subsection{Single Grain Deformation Modeling}

The fully anisotropic visco-plastic self-consistent (VPSC) polycrystal plasticity model was originally developed by Lebensohn and Tomé [1] and has seen continuous improvements since then. An updated comprehensive description of the method can be found in Lebensohn et al [29]. In brief: metallic materials are formed by crystallographic domains called grains (shown in the EBSD microscopy of Figure 3 with superimposed c-axis directions shown with black arrows). Within VPSC the metallic aggregate (polycrystal) is modeled by weighted orientations (crystals), representing a grain and its associated volume fraction as shown in the right image in Figure 3, respectively. The response and properties of such aggregate are given by averaging the response and the properties of the individual constituent grains (typically, between 1000 to 3000 grains are chosen to reproduce the texture). VPSC keeps track of the stress, strain, crystal orientation, activity and activation stress in each slip system in each grain, dislocation density on each slip system, etc.

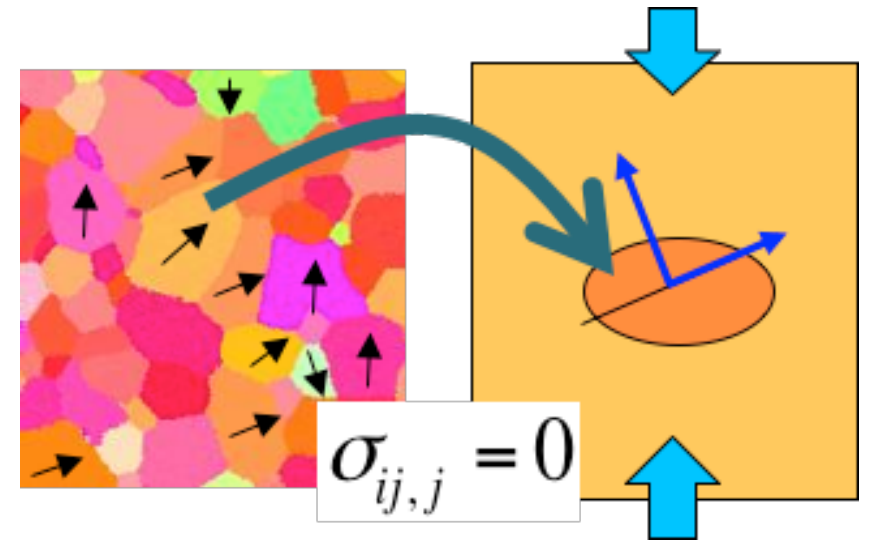

Figure 3: Schematic showing the representation of a grain and its surrounding environment as an ellipsoidal inclusion embedded in an effective medium. The interaction with the medium determines the stress and strain rate in the inclusion

VPSC treats each grain and its interaction with the surrounding environment as an ellipsoidal viscoplastic inclusion embedded in an effective visco-plastic medium. Both, inclusion and medium have fully anisotropic properties. The effective medium represents the 'average' environment 'seen' by each grain (Figure 3). When boundary conditions of stress or strain rate are applied to the effective medium, an interaction between medium and grain (embedded inclusion) takes place, and local stresses are induced in the inclusion and its vicinity, which drive the deformation of the inclusion. The stresses, which follow from solving the stress equilibrium equations, are uniform inside the ellipsoidal inclusion, but deviate from the average stress in the medium depending on the relative properties of medium and inclusion. The 'self consistency' of the approach arises from enforcing the condition that the average stress and strain rate over all the grains has to be equal to the boundary values imposed on the medium.

The propagation of slip dislocations induces plastic shear and no volumetric dilatation. Denoting $n^{s}$ the normal to the slip plane, and $b^{s}$ the direction of shear (parallel to the Burgers vector), the resolved shear stress on the shear plane and along the shear direction associated with the stress tensor acting on the grain is given $b^{1}$

$$
\tau_{r e s}^{s}=b_{i}^{s} n_{j}^{s} \sigma_{i j}^{c}=m_{i j}^{s} \sigma_{i j}^{c}
$$

\footnotetext{
${ }^{1}$ Unless stated otherwise, throughout this text the implicit summation convention is used: repeated indices in equations denote summation over the range of such indices.
} 
where

$$
m_{i j}^{s}=\frac{1}{2}\left(b_{i}^{s} n_{j}^{s}+b_{j}^{s} n_{i}^{s}\right)
$$

is the Schmid tensor that projects the stress along the shear plane. The strain rate tensor associated with shear rate $\dot{\gamma}^{s}$ in system $s$ is

$$
\dot{\epsilon}_{i j}^{c}=m_{i j}^{s} \dot{\gamma}^{s}
$$

The strain rate associated with climb of forest and loop dislocation segments is given by (Woo, 1985)

$$
\dot{\epsilon}_{i j}^{(\text {climb })}=\operatorname{sym}\left(\sum_{s} \rho^{s}\left(\hat{t}^{s} \otimes \bar{v}^{s}\right)_{i} b_{j}^{s}\right)
$$

where $\rho^{s}$ is the density of segments 's', $v^{s}$ is their climb velocity, $b^{s}$ is their Burgers vector, $\hat{t}^{s}$ is their tangent vector. In the case of climb only the edge component of the dislocation matters. If all dislocations are assumed to be pure edge dislocations, Equation 10 becomes

$$
\dot{\epsilon}_{i j}^{(\operatorname{climb})}=\operatorname{sym}\left(\sum_{s} \rho^{s} v^{s} b^{s} \hat{b}_{i}^{s} \hat{b}_{j}^{s}\right)
$$

The dislocation climb and glide systems considered in HCP Zr crystals represented in VPSC are depicted in Figure 4 [19]. The glissile dislocations are: $\{1010\}\langle 1120\rangle$ prism slip (3 systems), $\{0001\}\langle 1120\rangle$ basal slip (3 systems), $\{1122\}\langle 1123\rangle$ pyramidal slip (12 systems). Also, the one sessile dislocation loop (0002) $\langle 0002\rangle$ in the basal plane, and three sessile loops $\{1120\}\langle 1120\rangle$ in prism planes are represented in the model. The experimental observations indicate that basal loops present a vacancy stacking fault configuration, and prism loops an interstitial stacking fault configuration. It is known that the grain size effects on irradiation growth rate are non-negligible for zirconium alloys below a critical grain size of $\sim 8 \mu \mathrm{m}[30,31]$. These effects arise from the competition between grain boundaries and the intragranular dislocation structure in trapping defects. The crystal plasticity model described herein does not consider the sink strengths of grain boundaries for defects. Incorporation of grain boundary trapping is planned for future work.

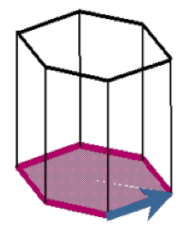

$(0001)\langle 11 \overline{2} 0\rangle$

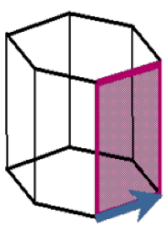

$(10 \overline{1} 0)\langle 11 \overline{2} 0\rangle$
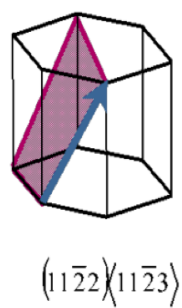
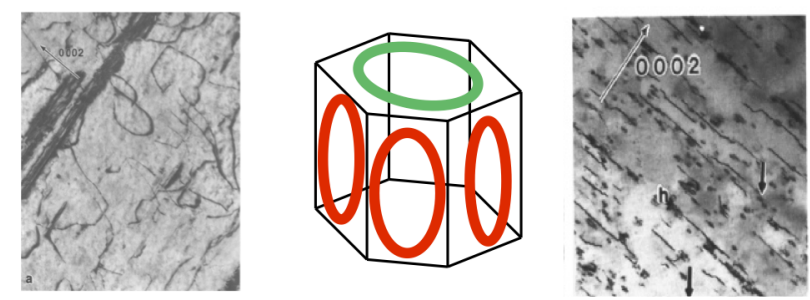

Figure 4: Basal, Prism and Pyramidal dislocations introduced by tube manufacturing process; microstructure of unirradiated Zrly-2; prism and basal dislocations loops introduced by irradiation; microstructure of irradiated Zrly-2 [19].

For describing creep, a visco-plastic constitutive law is used in which the shear rates are given by a power of the ratio between resolved shear and a threshold stress;

$$
\dot{\gamma}^{s}=\dot{\gamma}_{o}\left(\frac{m_{k l}^{s} \sigma_{k l}}{\tau_{\text {thres }}^{s}}\right)^{n}
$$

Here $\dot{\gamma}_{o}$ is a normalization factor and $n$ is the rate-sensitivity exponent. When several systems are active in a grain, the strain rate is given by the sum of their shear rates

$$
\dot{\epsilon}_{i j}=\sum_{s} m_{i j}^{s} \dot{\gamma}^{s}=\dot{\gamma}_{o} \sum_{s} m_{i j}^{s}\left(\frac{m_{k l}^{s} \sigma_{k l}}{\tau_{\text {thres }}^{s}}\right)^{n}
$$


Linearizing Equation 13 inside the domain of a grain gives:

$$
\dot{\epsilon}_{i j}=M_{i j k l}(\sigma) \sigma_{k l}+\dot{\epsilon}_{i j}^{o}+\dot{\epsilon}_{i j}^{\text {growth }}
$$

where $M_{i j k l}$ and $\epsilon_{i j}^{o}$ are the viscoplastic compliance and the back-extrapolated term for a given grain, respectively. The linearization (Equation 14) of the constitutive response is required to solve the equilibrium conditions between inclusion and medium, and is actually a tangent approximation to the non-linear law (Equation 13). The following sections demonstrate that irradiation creep, thermal creep, and instantaneous plasticity can each be expressed in the form of Equation 13.

In Equation 14 a stress-independent extra term is added in order to account for the irradiation growth rate of the grain. The constitutive law giving the response of the effective medium (polycrystal) is given by a linear relation analogous to Equation 14,

$$
\overline{\dot{\epsilon}}_{i j}=\bar{M}_{i j k l} \bar{\sigma}_{k l}+\overline{\dot{\epsilon}}_{i j}^{o}
$$

where $\bar{\epsilon}_{i j}$ and $\bar{\sigma}_{k l}$ are overall (macroscopic) magnitudes and $\bar{M}_{i j k l}$ and $\bar{\epsilon}_{i j}^{o}$ are the macroscopic viscoplastic compliance and back extrapolated rate, respectively. The growth of the polycrystal cannot be separated from the back-extrapolated parameter and the term $\overline{\hat{\epsilon}}_{i j}^{o}$ accounts for both. The macroscopic moduli are unknown a priori and need to be adjusted self-consistently by enforcing the condition that the stress and strain rate averaged over all grains has to be equal to the stress and strain rate of the effective medium

$$
\overline{\dot{\epsilon}}_{i j}=\left\langle\dot{\epsilon}_{i j}\right\rangle \quad \text { and } \quad \bar{\sigma}_{k l}=\left\langle\bar{\sigma}_{k l}^{c}\right\rangle
$$

\subsection{Representative Zirconium Alloy Texture}

The distribution of orientations of the crystals associated with each grain (the hexagonal cell shown in Figure 4) is called texture. Preferential orientations are called texture components, and are characteristic of the fabrication process (e.g. rolling, extrusion, torsion). The importance of texture is that the directional strength of texture components tends to dominate the mechanical response of the polycrystal and determines the anisotropy of the mechanical response. Different alloys and different manufacturing routes can lead to different textures which in turn lead to different creep and growth behavior during irradiation. As a consequence, introducing the texture explicitly in the modeling leads to variation in the macroscopic response - although the constitutive response of the constituent grains is the same - and allows for representation of anisotropic behavior.

In the specific case of zirconium alloy cladding tubes, the constituent grains have hexagonal symmetry, characterized by a unit cell with the c-axis perpendicular to the compact basal plane. Hexagonal crystals exhibit highly anisotropic mechanical behavior, exhibiting very different properties along the c-axis or perpendicular to it. A specific example is the irradiation-induced formation of interstitial loops in the prism planes, while excess vacancies form loops on the basal planes[5]. This configuration of loops is responsible for negative growth along the c-axis and positive growth perpendicular to it. The extrusion-forming of the cladding tube orients the c-axis of these crystals mostly in the radial(RD)-hoop(HD) planes (Figure 5), with two maxima at about $\pm 30^{\circ}$ from the radial axis. As a consequence, prism planes are oriented mostly along the axial directions( $\mathrm{AD})$, which explains the positive axial growth observed in cladding.

As mentioned in Section 2.1 the texture is represented by a discrete number of grains and, internally, VPSC keeps track of several state variables inside each grain (stress, strain, crystal orientation, slip activity and activation stress). While this modus operandi is feasible when using VPSC in s stand-alone mode, it leads to onerous run time and memory storage requirements when VPSC is used to describe the mechanical response at each integration point in an engineering scale finite element simulation required for fuel performance analysis. As a consequence, the strategy chosen in this work is to optimize run times in the fuel behavior code by finding a compromise that minimizes the number of grain orientations used to represent the texture, while preserving the accuracy of the response calculated by VPSC.

The role of texture in altering the macroscopic response is well recognized and it has been introduced in the continuum models of creep and growth through the use of Kearns factors[32]. The latter utilize a quantitative measure of the basal component (c-axis orientation) in the texture to 'weight' the directional 


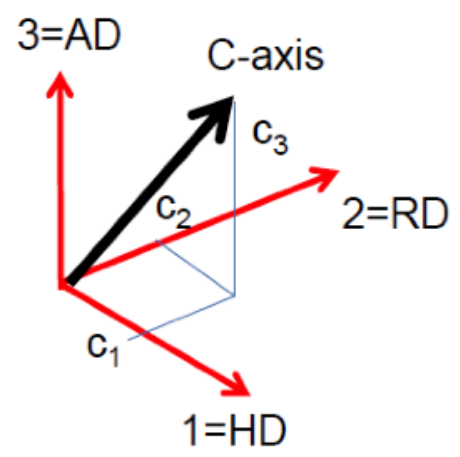

Figure 5: Definition of the Crystallographic Orientation of a Zirconium Grain Based on C-axis Direction

response of the cladding tube. As shown in Figure 5, if $\hat{c}=\left(c_{1}, c_{2}, c_{3}\right)$ are the components of the c-axis with respect to tube axes [Hoop $(1=\mathrm{HD})$, Radial $(2=\mathrm{RD})$, and Axial $(3=\mathrm{AD})$ ], the Kearns factors $\left(f_{i}\right)$ are defined as the average of the projections of the c-axis (direction cosines) of the $N_{g}$ grains that define the polycrystal. This is given by,

$$
f_{i}=\frac{1}{N_{g}} \sum_{g=1}^{N_{g}}\left(c_{i}\right)^{2} \quad(i=1,2,3)
$$

For the cladding tube material texture used in this work, measurements from a representative cladding tube sample were obtained using X-ray Diffraction (XRD) techniques and it was determined that depicting 1944 grains in VPSC suffice to fully capture the crystallographic texture. The associated measured Kearns factors for the cladding tube material are 0.424 (hoop), 0.547 (radial) and 0.028 (axial) (see Table 1). In addition, models containing a reduced number of grains to represent the texture were generated using approximately the same Kearns factors. Figure 6 shows the basal plane pole figure texture map generated from the 1944 grain model and the three texture maps produced by the reduced 100-, 4- and 2-grain models. In all cases, the texture displays a predominate crystal orientation with the basal poles oriented perpendicular to the axial (rolling) direction and $\pm 30^{\circ}$ from the radial direction.

The results from irradiation growth and creep calculations extending out to 6 dpa find that the results from the 100-grain model are nearly indistinguishable from that of the 1944-grain model using the texture parameters shown in Table 1; while the 4-grain and 2-grain models yield deviations in the irradiation growth strains of about 5\% and 20\%, respectively. Based on this evaluation, the 4-grain texture represents an acceptable compromise between accuracy and computation time. Further work to interface the VPSC model into BISON used the 4-grain model for testing and evaluation purposes.

Table 1: Kearns factor for different numbers of reduced grains representing the Zirconium alloy texture. $\mathrm{X}=$ direction 1 (hoop), $\mathrm{Y}=$ direction 2 (radial), $\mathrm{Z}=$ direction 3 (axial)

\begin{tabular}{|c|c|c|c|c|c|}
\hline $\begin{array}{c}\text { Number of } \\
\text { Grains }\end{array}$ & $\mathbf{f}_{\mathbf{x}}$ & $\mathbf{f}_{\mathbf{y}}$ & $\mathbf{f}_{\mathbf{z}}$ & $\begin{array}{c}\text { Difference in } \\
\text { Growth Strain }\end{array}$ & Runtime (sec) \\
\hline 1944 & 0.4244 & 0.5472 & 0.0284 & 0.0 & 2012 \\
\hline 100 & 0.4178 & 0.5516 & 0.0306 & 0.0 & 118 \\
\hline 4 & 0.4244 & 0.5472 & 0.0284 & $5 \%$ & 19 \\
\hline 2 & 0.3290 & 0.6710 & 0.0 & $20 \%$ & 18 \\
\hline
\end{tabular}




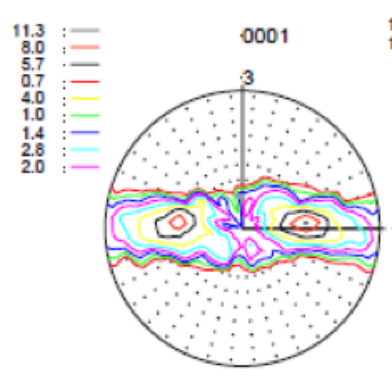

1944 grains

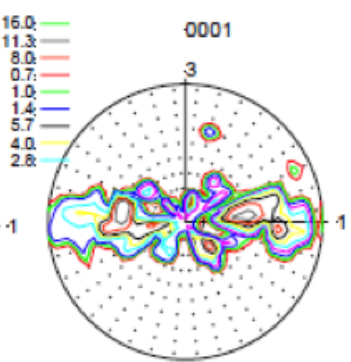

100 grains

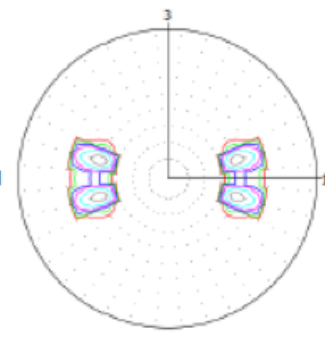

4 grains

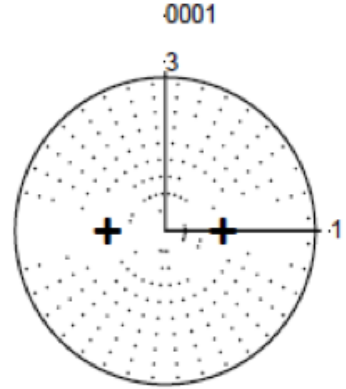

2 grains

Figure 6: Cladding tube texture representation using different number of orientations (1:hoop, 2:radial; 3:axial direction)

\subsection{Irradiation Growth and Irradiation Creep}

Neutron irradiation produces vacancies, interstitial atoms and interstitial clusters in irradiated Zr alloys, with concentrations far greater than their thermal equilibrium concentrations. These defects eventually get absorbed by sinks, such as dislocations and dislocation loops, and induce dimensional changes in the material. When dislocations preferentially absorb vacancies or interstitial atoms, they climb. Dislocation climb may be followed by glide (climb assisted glide) in the presence of a resolved shear stress. The associated dimensional changes are called irradiation growth and irradiation creep, respectively, and are driven by a high concentration of irradiation-produced defects.

The two phenomena of irradiation creep and irradiation growth are coupled in polycrystalline materials. This is because the growth of a grain induces reaction stresses that result from the grain interaction with its neighbors (which are also growing but generally not at the same directional rate). These stresses, in turn, induce irradiation (and possibly thermal) creep in the grains, even if at the macroscopic level the material is stress-free. Under some non-standard operating conditions, internal stresses may be high enough to activate thermal creep, which helps relax the internal stress. A distinguishing feature of the polycrystal approach used in VPSC is that it accounts explicitly for these internal stresses and the coupling of creep and growth mechanisms, a feature that is absent in empirical continuum models used in fuel behavior calculations. The latter rely on an independent linear superposition of these regimes, expressed as a function of the macroscopic temperature and stress boundary conditions.

Recently, an irradiation growth model for single crystal zirconium developed by Golubov and Barashev has been incorporated into the VPSC framework to capture the atomic processes controlling single crystal growth[33]. The most relevant feature of Golubov's model is to postulate that the interstitial clusters created during neutron irradiation are 1-D diffusers on the basal plane, while vacancies and interstitial atoms are $3 \mathrm{D}$ diffusers $[34,35,36]$. By requiring this behavior to be consistent with irradiation growth experiments performed by [37] on single crystals, Golubov et al deduce empirically a rate of nucleation of dislocation loops with dose. Interstitial loops in the Golubov model start nucleating on prism planes early in the irradiation process, while the model calculates that the nucleation of vacancy loops on basal planes are delayed. The calculated nucleation rate vs dose and evolution of loops radius vs dose is illustrated in Figure 7.

Incorporating Golubov et al's reaction-diffusion model into the VPSC framework required generalization to a full crystallographic representation, including recent advances by Golubov. et al. to include the mechanism of Climb-Controlled Glide (CCG) to account for creep effects. In Equation 10, the climb rate is a function of dislocation climb velocity. For edge dislocations and loops, the climb velocity is given by the difference between the number of interstitial atoms and the number of vacancies absorbed by the dislocation $s$ per unit time and unit length: $v^{s}=\dot{N}_{i \rightarrow s}-\dot{N}_{v \rightarrow s}$. The single crystal model of Golubov et al [35] provides these rates for the basal plane edge dislocation (c-direction Burgers vector) and the prismatic plane dislocations $\left(\mathrm{a}_{1}, \mathrm{a}_{2}\right.$, and $\mathrm{a}_{3}$ Burgers vector) as a function of the irradiation dose, which can be used in VPSC. However, the eighteen glissile dislocation systems (3 basal, 3 prism, 12 pyramidal) considered in 


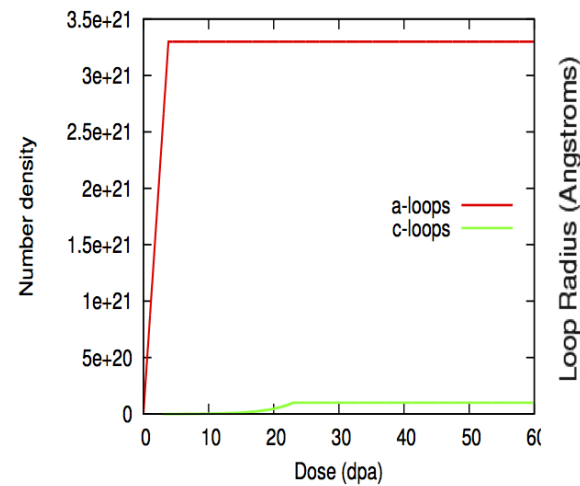

(a)

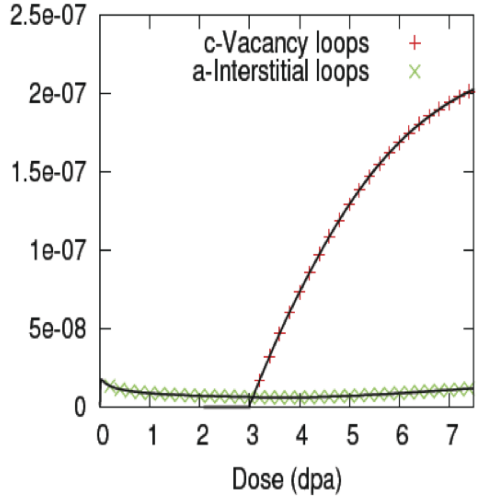

(b)

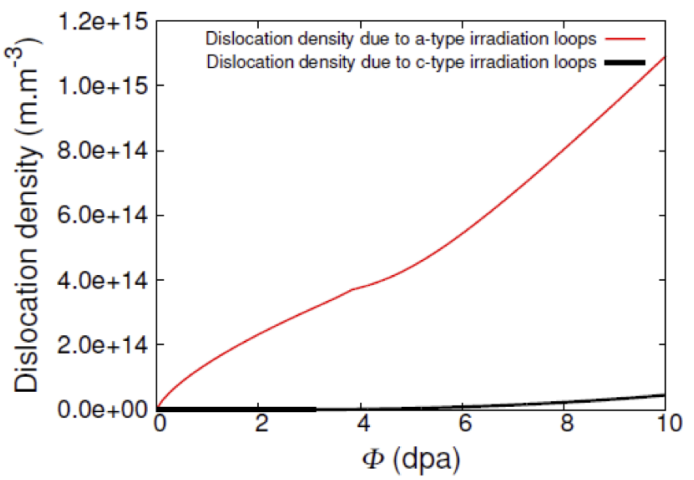

(c)

Figure 7: (a) Rate of loop nucleation proposed by Golubov et al [34]; (b) predicted evolution of average loop radius vs dose (red: interstitial prism loops; green: vacancy basal loops); (c) Calculated change of loop density as a function of neutron dose (expressed as displacements per atom, dpa) by the Golubov model.

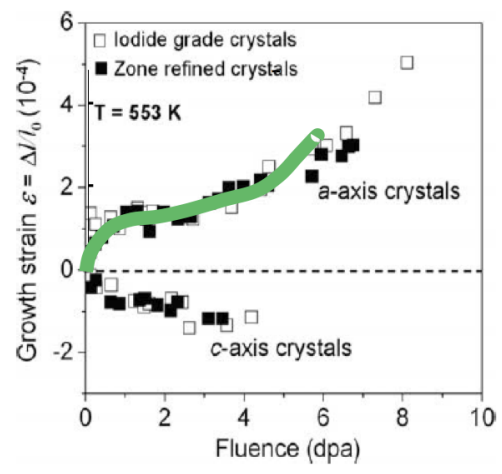

Figure 8: experimental growth measured by Rogerson and Zee [37] in single crystal and evolution growth predicted by Golubov, et al.[35].

VPSC have to be projected along the four glide directions provided by the Golubov model. Assuming the 
density is equal in all dislocation systems that belong to each mode, the conversion law is:

$$
\begin{aligned}
& \rho_{a_{i}}^{g l i s}=\rho^{\text {bas }}+\rho^{\text {pri }}+\rho^{\text {pyr }} \quad(i=1,3) \\
& \rho_{c}^{g l i s}=9 \rho^{\text {pyr }}
\end{aligned}
$$

The sessile a-loops (3) and c-loop (1) each exhibit a Burgers vector, and their respective densities are given by $\rho_{a_{i}}^{\text {loop }}:(i=1,3), \quad \rho_{c}^{\text {loop }}$. Equation 10 provides the strain rate for the climb of the edge component of the glissle dislocation systems $(s)$ and the climb of the sessile dislocation loops $(l)$ :

$$
\dot{\epsilon}_{m n}^{\text {growth }}=\frac{1}{2} \sum_{s=1}^{N_{s}} v^{s} \rho^{s}\left(b_{m}^{s} b_{n}^{s}+b_{n}^{s} b_{m}^{s}\right)+\frac{1}{2} \sum_{l=1}^{N_{l}} v^{l} \rho^{l}\left(n_{m}^{l} n_{n}^{l}+n_{n}^{l} n_{m}^{l}\right)
$$

where the edge dislocations are separated from the loop contributions, and the Burgers vector of the loop is collinear with the normal to the loop.

To incorporate irradiation creep into the formulation, the relatively simple climb-controlled-glide model of Gittus is adopted[38, 39]. In this model the shear rate of a dislocation segment is proportional to the resolved shear stress acting on the segment and inversely proportional to the time it takes to climb over obstacles. The latter is taken here as the average radius of loops $r_{\text {mean }}^{l}$ divided by the climb velocity $v^{s}$. The shear motion contributed by a dislocation system is also proportional to the availability (density) of segments:

$$
\dot{\gamma}^{s}=\rho^{s} \frac{m_{k l}^{s} \sigma_{k l}}{\mu} \frac{v^{s}}{r_{\text {mean }}^{l}}
$$

and the total creep that results from all dislocations that climb-glide is:

$$
\dot{\epsilon}_{i j}^{\text {creep }}=\sum_{s=1}^{N} m_{i j}^{s} \dot{\gamma}^{s}=\sum_{s=1}^{N} m_{i j}^{s} \rho^{s} \frac{m_{k l}^{s} \sigma_{k l}}{\mu} \frac{v^{s}}{r_{\text {mean }}^{l}}
$$

where $\sigma_{k l}$ is the stress in the grain; $m_{i j}^{s}$ is the Schmid tensor that projects the stress onto the slip plane of system $\mathrm{s} ; \rho_{s}$ is the glissile dislocation density for this system; $v^{s}$ is the climb velocity of the glissile dislocations; $\mu$ is the shear modulus. Observe that Equation 21 is a particular case of Equation 13 with creep exponent $n=1$. The sum of growth and creep rates given by Equations 19 and 21 is of the general form of the strain rate that enters in the VPSC formulation (Equation 14),

$$
\dot{\epsilon}_{i j}=\dot{\epsilon}_{i j}^{c r e e p}+\dot{\epsilon}_{i j}^{\text {growth }}=M_{i j k l}(\sigma) \sigma_{k l}+\dot{\epsilon}_{i j}^{o}+\dot{\epsilon}_{i j}^{\text {growth }}
$$

and this formulation is applied in the VPSC model to predict the dimensional changes of a cladding tube subjected to irradiation and stress.

\subsection{Thermal Creep}

Thermal creep takes place by glide of dislocations overcoming obstacles via stress and temperature activated mechanisms of diffusion. As such, it is relevant at high temperatures $\left(T>0.5 T_{m}\right)$ or at applied stresses approaching the flow stress $\left(\sigma>0.75 \sigma_{f}\right)$. While thermal creep is not a major player under normal reactor operating conditions, it becomes important under abnormal or accident conditions, where localized temperature and stress rises can take place. The viscous power law expressed by Equation 13 is appropriate for describing the contribution of several slip systems to the thermal creep in a grain. By defining the rate sensitivity exponent applicable to thermal creep, Equation 13 becomes;

$$
\dot{\epsilon}_{i j}^{\text {thermal }}=\sum_{s} m_{i j}^{s} \dot{\gamma}^{s}=\dot{\gamma}_{\circ}(T) \sum_{s} m_{i j}^{s}\left(\frac{m_{k l}^{s} \sigma_{k l}}{\tau_{\text {thres }}^{s}}\right)^{4}
$$

As in the case of irradiation creep, glide is assumed to occur along the prism, basal and pyramidal planes, hence the summation over $s$. The parameters that enter into Equation 23 are related to physical properties 
of $\mathrm{Zr}$ alloys. The parameters used in VPSC at this time were derived by Christodoulou et al [40] from experiments done on $\mathrm{Zr}-2.5 \% \mathrm{Nb}$ alloy tubes, at different temperatures and imposing various stresses along different directions. The experiments of Christodoulou et al suggest an interval of $3.8<n<4.2$ for the rate sensitivity parameter. A rate sensitivity parameter $(n)$ equal to 4 is used for this application. The threshold stresses that appear in Equation 23 that fit the experimental data are equal to 0.100, 0.111, and 0.300 GPa for prism, basal and pyramidal glide, respectively.

The reference shear stress $\dot{\gamma}_{\circ}$ depends on temperature and the activation energy for the diffusion controlled mechanism. The authors make the simplifying assumption that $\dot{\gamma}_{0}$ is the same for all systems while assigning a different activation threshold value $\tau_{\text {thres }}^{s}$ to each system. Christodoulou et al report that the reference shear stress can be represented by:

$$
\dot{\gamma}_{\circ}(T)=\dot{\gamma}_{\circ}(T=523) \exp \left[-Q(T)\left(\frac{1}{T}-\frac{1}{523}\right)\right]
$$

where $\dot{\gamma}_{\circ}(T=523)=4.157 \times 10^{-3} h^{-1}$ and $Q(T)=\left(5000+\frac{5600}{1+\exp ^{-(T-470) / 15}}\right)$

Observe that the pre-exponential factor in Equation 24 varies with temperature, suggesting that the character of the activation mechanism may be changing with temperature. Since the temperature interval covered by the experiments is between $373 \mathrm{~K}$ and $596 \mathrm{~K}$, Equation 24 may not be appropriate for simulating accident conditions (such as high temperature tube ballooning, discussed in Section 4). The applications of the thermal creep model are presented in Section 2.6, where all deformation mechanisms are combined to yield an overall deformation rate, and in Section 4, where a simulation of ballooning is presented.

\subsection{Instantaneous Plasticity}

Instantaneous plasticity is distinguished from creep in that the former is present when the stress is close to the flow stress or when a deformation rate is imposed. Abnormal conditions in an operating nuclear reactor can be caused by temperature fluctuations associated with loss of coolant accident (LOCA) or reactivity initiated accident (RIA). Increases in temperature, internal cladding pressure, and stress can locally induce instantaneous plastic deformation and lead to ballooning and burst of cladding tubes. Mobile dislocations will interact with irradiation produced microstructure (e.g. interstitial clusters or dislocation loops). The effect of irradiation on flow stress and strain hardening is increased yield strength and decreased ductility with increasing irradiation dose [41, 42, 43, 44, 45]. Irradiation inhibits twinning [44] and, in addition, twinning is not active in Zircaloy above 400K. As a consequence, twinning is not considered in cladding behavior applications.

The maximum value of internal stress that the material can sustain is the flow stress, a state where dislocations are emitted, resulting in a limiting stress condition. During plastic deformation or during irradiation the dislocation density increases, and with it the density of defects that the migrating dislocations have to overcome. As a consequence, the flow stress is increased both with deformation and irradiation, a phenomenon known as hardening (work or irradiation). A dislocation evolution model of hardening proposed by Beyerlein and Tomé [27] for Zr is implemented in VPSC along with a recent addition of dislocation loop evolution associated with irradiation. The parameters of the model are adjusted to experimental data obtained by Le Saux et al [42] on irradiated and un-irradiated Zircaloy-4 tested in tension at different temperatures and rates. The approach accounts for glide of dislocations in the prismatic, basal and pyramidal planes. The total strain rate in the grain is given by the expression contained in Equation 13 with an appropriate rate sensitivity parameter $(n=20)$ that ensures that no deformation is activated unless the resolved shear stress is near the threshold shear stress.

$$
\dot{\epsilon}_{i j}^{\text {plastic }}=\sum_{s} m_{i j}^{s} \dot{\gamma}^{s}=\dot{\gamma}_{\circ} \sum_{s} m_{i j}^{s}\left(\frac{m_{k l}^{s} \sigma_{k l}}{\tau_{\text {thres }}^{s}(\dot{\epsilon}, T)}\right)^{20}
$$

The threshold stress $\tau_{\text {thres }}^{s}$ shown in Equation 25 is a function of strain rate and temperature, as described by Beyerlein and Tomé [27]. The dependence of the threshold stress on dislocation density is given by the 
classical Taylor work hardening law formulation[46];

$$
\tau_{\text {thres }}^{s}=\tau_{\circ}^{s}+\alpha^{f} \mu b^{s} \sqrt{\rho^{f}}
$$

where the index $f$ refers to total density of forest dislocations, $b^{s}$ is the Burgers vector of system $s$, and the dislocation interaction parameter $\left(\alpha^{f}\right)$ varies between 0.1 and 1.0 for this application. The total dislocation density defines a mean free path for dislocations. The effect on hardening from irradiation induced sessile dislocations loops is treated separately, and is explained below. The dislocation density evolution in each system is composed of a storage term $\left(k_{1}\right)$ and an annihilation term $\left(k_{2}\right)$ as shown below:

$$
\frac{\delta \rho^{s}}{\delta \gamma^{s}}=k_{1} \sqrt{\rho^{s, f}}-k_{2}(\dot{\epsilon}, T) \rho^{s, f}
$$

The material parameters for the constitutive model were determined using a fit against the experimental uniaxial tensile stress-strain data reported by Le Saux et al. for unirradiated Zircaloy-4 material[42]. The annihilation coefficient $k_{2}$ in Equation 27 depends on an activation energy and a drag stress specific to each dislocation mode. As a consequence, the evolution of prism, basal and pyramidal dislocations is not only a function of temperature but also different for each one. For guidance, and to give an idea of plastic anisotropy, the values of $\tau_{\circ}^{s}$ for prism, basal, and $<\mathrm{c}+\mathrm{a}>$ pyramidal dislocations in Equation 26 are 91, 243, $320 \mathrm{MPa}$, respectively, at $\mathrm{T}=553 \mathrm{~K}$.

The polycrystal material is represented using 1944 crystal orientations. The initial crystallographic texture used is the one shown in Figure 6 and corresponds to a cladding tube. The curve fits, along with the data reported by La Saux et al. for $280^{\circ} \mathrm{C}<\mathrm{T}<480^{\circ} \mathrm{C}$ and $0.01 s^{-1}<\dot{\epsilon}<5 s^{-1}$ are shown in Figure 9

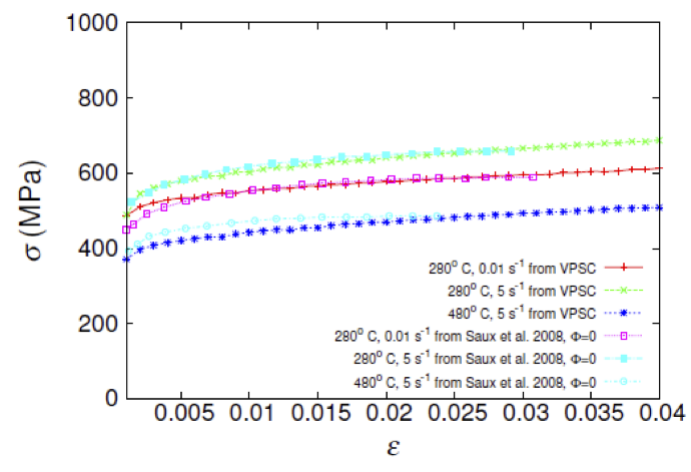

(a)

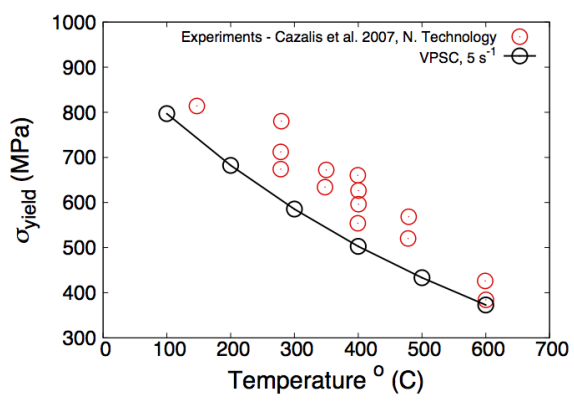

(b)

Figure 9: (a) predicted stress-strain response at different strain rates and temperatures for an unirradiated Zr-4 tube along the axial direction, superimposed with data from Le Saux et al[42] used for fitting hardening model parameters; (b) predicted yield stress at different temperatures for $5 \mathrm{~s}^{-1}$ rate, and comparison with Zircaloy-4 data of Cazalis et al. [45]

Next, the effect of irradiation-induced microstructure evolution on hardening of the material is incorporated into the threshold stress by accounting for resistance to slip of immobile dislocation loops. The interaction of forest dislocations with immobile dislocation loops in basal and prism planes has the same dependence as in Equation 26 [41, 47, 48], except that the interaction constant adopts a different value:

$$
\tau_{\text {thres }}^{s}=\tau_{\circ}^{s}+\alpha^{f} \mu b^{s} \sqrt{\rho^{f}}+\alpha^{l} \mu b^{s} \sqrt{\sum_{l} N^{l} 2 \pi r_{\text {mean }}^{l}}
$$

Here, $r_{\text {mean }}^{l}$ is the average loop radius and $N^{l}$ is the number of loops of type $l$ per unit volume. The evolution of loop radius and density in each grain follows from the growth model of $[34,36]$ and its interface with VPSC described in Section 2-3 above. At any instance of an irradiation simulation, the boundary conditions may be modified to allow for temperature and/or stress changes which may lead to the activation 
of this instantaneous plasticity mechanism. Figure $7 \mathrm{c}$ shows an example of the loop density as a function of irradiation dose as calculated by the Golubov model.

In Figure 10, tensile axial tests at $280^{\circ} \mathrm{C}$ for irradiated cladding are compared to the VPSC analysis using the 1944 grain model. Two tensile rates were considered: $\dot{\epsilon}=0.01 \mathrm{~s}^{-1}$ (Figure 10a) and $\dot{\epsilon}=5 \mathrm{~s}^{-1}$ (Figure 10b). Figure 10c compares the calculated variation in yield stress as a function of fast neutron dose for the case of $\dot{\epsilon}=0.01 \mathrm{~s}^{-1}$ along with the experimental data for the irradiated yield stress reported by Le Saux et al. for Zircaloy-4 at $\sim 10$ dpa. The experimental results indicate that irradiation dose of $10 \mathrm{dpa}$ increases substantially the flow stress compared to unirradiated cladding. For the comparisons shown in Figure 10, it is assumed that the conversion factor from fast fluence $(>1 \mathrm{MeV})$ to displacement per atom (dpa) is $1 \times 10^{25} \mathrm{n} / \mathrm{m}^{2} \approx 1 \mathrm{dpa}$.

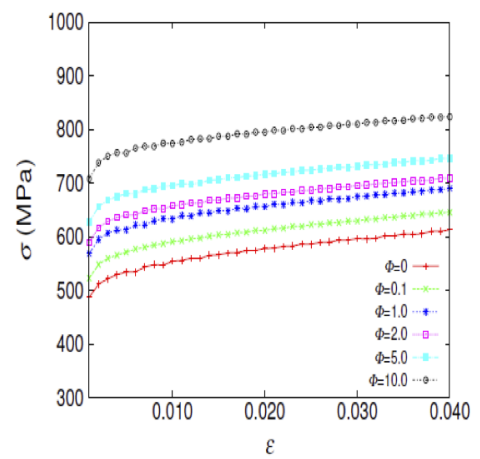

(a)

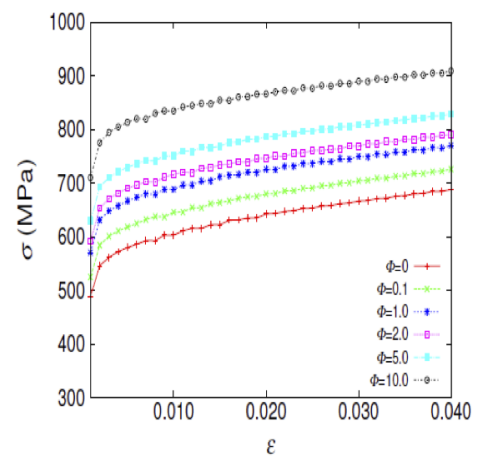

(b)

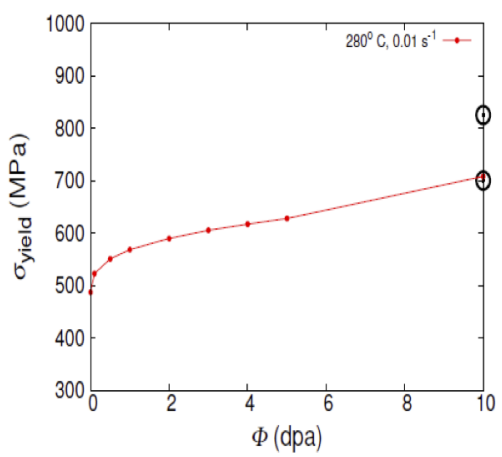

(c)

Figure 10: Simulations of tensile axial tests done at $280^{\circ} \mathrm{C}$ on cladding pre-irradiated to $10 \mathrm{dpa}$. (a) tensile rates of $\dot{\epsilon}=0.01 s^{-1}$; (b) tensile rate of $\dot{\epsilon}=5 s^{-1}$; (c) variation of yield stress versus dose for the case $\dot{\epsilon}=0.01 s^{-1}$, and experimental data (circles) reported by Le Saux et al (2008) for Zircaloy-4 irradiated to $10 \mathrm{dpa}$.

From the results of this section it follows that a work and irradiation hardening model based on the evolution of dislocation density is able to capture the temperature and rate-dependent stress-strain response of non-irradiated Zircaloy-4, and also the effect of irradiation-induced microstructure changes (irradiation damage) on the flow stress. The latter is captured through the increase of the threshold stress associated with the interaction of mobile network dislocations and immobile irradiation loops. No attempt is made here to introduce explicitly the effect on hardening of other microstructural features, such as grain size or precipitates or Laves phases. Those effects are indirectly accounted for when adjusting the parameters of the dislocation based hardening model using experimental flow stress curves.

\subsection{Stand-alone VPSC Results / Superposition 8 Coupling}

The following demonstrates the capability of VPSC to account for the superposition and coupling of the cladding deformation mechanisms caused by stress and irradiation, and provides a benchmark case for testing the interface between VPSC and the fuel behavior code, BISON. As suggested by the diagram in Figure 2, a given deformation regime will prevail depending on the neutron damage accumulation, stress, and temperature, acting on the cladding tube. However, these mechanisms do not exclude each other and, most importantly, the boundary conditions on the cladding may change during operation requiring that all the mechanisms need to be available, even if their contribution is negligible for the current set of boundary conditions. As a consequence, the constitutive law for the grain will be the sum of irradiation growth (Equation 19), irradiation creep (Equation 21), thermal creep (Equation 23) and instantaneous plasticity (Equation 25) contributions: 


$$
\begin{aligned}
\dot{\epsilon}_{m n}^{\text {total }} & =\frac{1}{2} \sum_{s=1}^{N_{s}}\left(\dot{N}_{i \rightarrow s}-\dot{N}_{v \rightarrow s}\right) \rho^{s}\left(b_{m}^{s} b_{n}^{s}+b_{n}^{s} b_{m}^{s}\right) \\
& +\frac{1}{2} \sum_{l=1}^{N_{l}}\left(\dot{N}_{i \rightarrow l}-\dot{N}_{v \rightarrow l}\right) \rho^{l}\left(n_{m}^{l} n_{n}^{l}+n_{n}^{l} n_{m}^{l}\right) \\
& +\dot{\gamma}_{\circ}(T) \sum_{s=1}^{N_{s}} m_{i j}^{s}\left(\frac{m_{k l}^{s} \sigma_{k l}}{\tau_{c r e e p}^{s}}\right)^{4}+\dot{\gamma}_{\circ} \sum_{s=1}^{N_{s}} m_{i j}^{s}\left(\frac{m_{k l}^{s} \sigma_{k l}}{\tau_{\text {inst }}^{s}(\dot{\epsilon}, T)}\right)^{20}
\end{aligned}
$$

The demonstration case chosen here to illustrate the VPSC capabilities is a pressurized cladding tube, subjected to the following piece-wise loading history:

$$
\begin{aligned}
& \sigma_{\text {axial }}=100 M P a \rightarrow 0 d p a<\Phi<13 d p a \\
& \sigma_{\text {axial }}=200 M P a \rightarrow 13 d p a<\Phi<20 d p a \\
& T=500 K \rightarrow 0 d p a<\Phi<7 d p a \\
& T=600 K \rightarrow 7 d p a<\Phi<20 d p a
\end{aligned}
$$

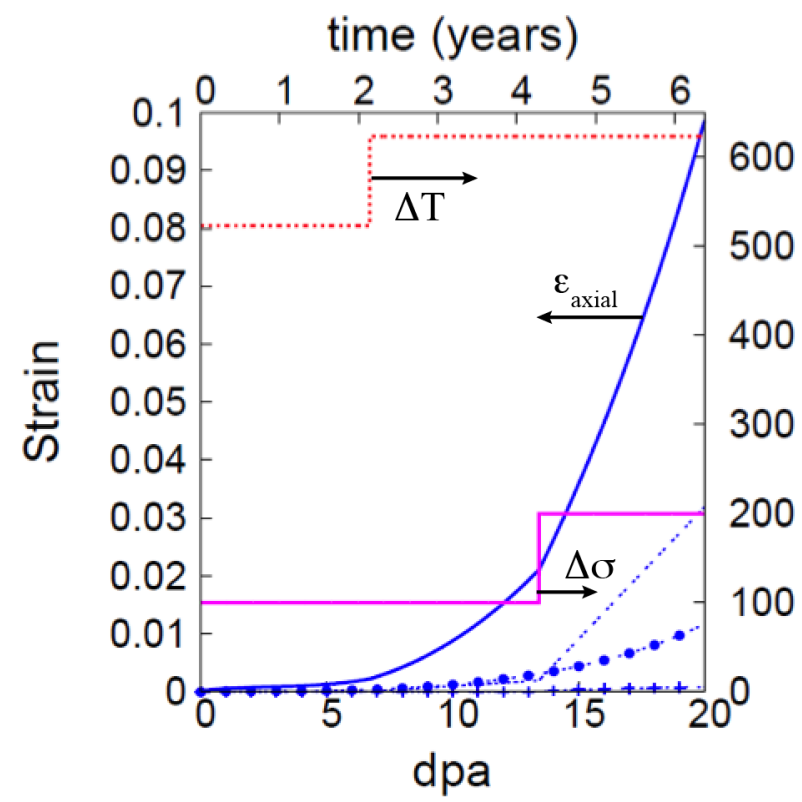

(a)
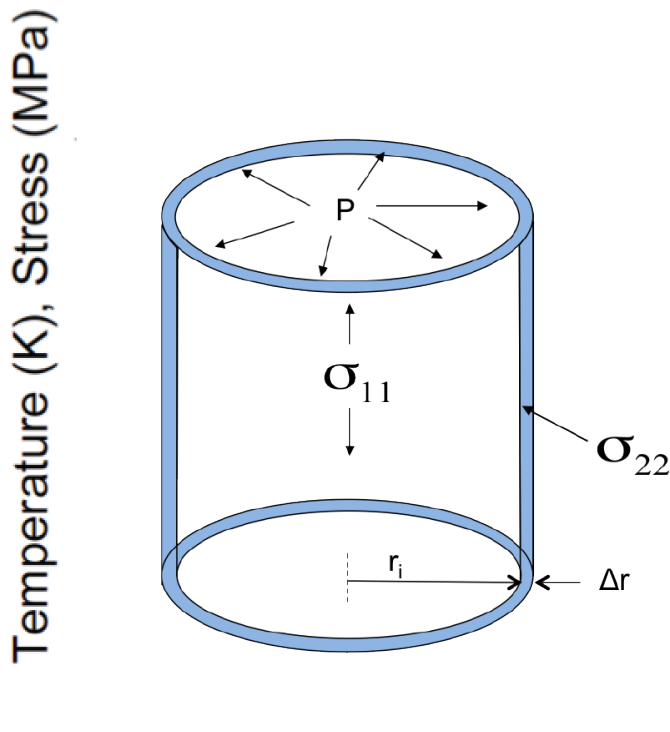

(b)

Figure 11: (a) Axial strain components as a function of fast neutron dose for a cladding tube subjected to the loading history Equation 30. (b) Schematic of the cladding tube geometry with the loading conditions defined. The line types in (a) identify the contribution to strain of each mechanism: total axial strain (blue solid line), the irradiation growth strain (blue $\bullet \bullet \bullet$ line), the thermal creep (blue dashed line), and the instantaneous plasticity (blue ++++ line)

For comparison purposes, the loading history is applied first with irradiation growth, thermal creep, and instantaneous plasticity strains active separately during the analysis. Second, an analysis has been performed in which all three forcing conditions are simultaneously active. Only the results of the latter case will be discussed. The evolution of the total axial strain with neutron dose (dpa) is depicted in Figure 11. Shown is the the total axial strain (solid line), the irradiation growth strain (dashed line with circles), the thermal creep (dashed line), and the instantaneous plasticity (dashed line with plus signs). The most 
important result is the observation that the axial strain resulting from the coupled mechanisms is not equal to the superposition of the individual mechanisms. Second, the irradiation effects become noticeable only after accelerated irradiation growth takes place at about $5 \mathrm{dpa}$. Third, the $100 \mathrm{~K}$ temperature increase at $7 \mathrm{dpa}$ has almost no effect on the thermal creep contribution until the stress increase at 13 dpa makes this mechanism relevant to the total deformation. Finally, the contribution of the instantaneous plasticity mechanism is negligible for the load conditions used in this evaluation.

\section{Interfacing a Microstructurally-Aware Constitutive Model with an Engineering Code}

The previous section described the mechanistic constitutive model contained within VPSC to calculate the polycrystalline response of zirconium alloys to imposed stress, temperature, and radiation environments. VPSC is a point model, and when used in standalone mode, the results represent material behavior under spatially uniform time-dependent temperature and irradiation conditions. In contrast, the thermal, mechanical, and radiation environments are strongly non-uniform within a fuel rod, and this non-uniformity influences the overall deformation behavior of the fuel rod. These conditions require fuel behavior analyses to consider both the spatial and time variations within the components, such as temperature gradients in the cladding, to properly capture the overall response. To utilize VPSC within the engineering scale fuel rod behavior code, BISON, a methodology is required to interface the single point constitutive model with the stress and strain analysis performed throughout the cladding at the macroscale. The approach reported herein will be to integrate VPSC into the finite element formulation used in BISON by calling the module at each integration point. Unlike the engineering-scale empirical correlations used in the current fuel performance codes, the computational cost of using the polycrystal constitutive law could be high depending on the level of detail that the material property model intends to capture. The advantage, on the other hand, is that the polycrystal-based constitutive law is general and independent of both the specific mechanical tests used to develop the model parameters and the macroscopic boundary conditions used in the finite element formulation.

The following describes the numerical procedure to implement VPSC within the finite element framework in the MOOSE system using a Newton-Raphson method and the results from a set of benchmark cases to test the functionality of the BISON/VPSC interface.

\subsection{Computational Methodology}

The finite element solution of a mechanical load and displacement problem involves solving the weak-form equation of the mechanical equilibrium condition. In the MOOSE system, the global residual associated with the weak-form equation is minimized using a variant of the Newton iteration method. To solve the nonlinear deformation problem involving creep and plasticity, the increment in total strain at each time step is passed to a material model which computes a trial stress tensor, and the trial stress tensor is then used to update the Newton iteration solution until the convergence is reached, i.e., the weak-form equilibrium equation is satisfied and the strain tensor consisting of the elastic strain and plastic strain is consistent with the applied stress. Such a computational process requires the VPSC module to compute a stress tensor for the increment in total strain. The calculation of the stress at each integration point involves a second, local Newton iteration to solve for the trial stress tensor. The VPSC to BISON embedded iteration is referred to as the sub-Newton iteration to distinguish this iteration method from the iteration method used in the MOOSE system solving the weak-form equation. A more detailed description of the computational method used to couple VPSC with a finite element code can be found in Reference [49].

At each time step, knowing the old stress tensor at time $t$, the difficulty is to provide an update of the trial stress at current time $t+\Delta t$ as shown in following equations.

$$
\begin{gathered}
\sigma_{t+\Delta t}=\sigma_{t}+C \Delta \epsilon_{e} \\
\Delta \epsilon_{e}=\Delta \epsilon_{\text {total }}-\Delta \epsilon_{p}
\end{gathered}
$$

where; 
$\sigma_{t}$ is the old stress tensor at time $\mathrm{t}$,

$C$ is the elastic stiffness tensor of the polycrystal

$\Delta \epsilon_{e}$ is the incremental elastic strain at time $\mathrm{t}$

$\Delta \epsilon_{\text {total }}$ is the total incremental strain at time $\mathrm{t}$

$\Delta \epsilon_{p}$ is the incremental plastic strain computed in VPSC including creep, irradiation growth, and instantaneous plasticity

The residual, $R$, is defined as the difference between the sum of the incremental elastic strain (as computed using the trial stress and the elastic stiffness) and plastic strain, and the total strain increment computed in the MOOSE system. The residual is calculated by;

$$
R=C^{-1} \Delta \sigma+\Delta t \cdot \dot{\epsilon}_{p}-\Delta \epsilon_{t}
$$

where;

$\Delta \sigma$ is given by $\sigma_{t+\Delta t}-\sigma_{t}$

$\Delta \epsilon_{t}$ is the total incremental strain computed in the MOOSE system

$\Delta t \cdot \dot{\epsilon}_{p}$ is equal to $\Delta \epsilon_{p}$

The Newton iteration method is used to compute the trial stress as shown below;

$$
\Delta \sigma_{k+1}=\Delta \sigma_{k}-\frac{\delta R}{\delta \Delta \sigma_{k}} R\left(\Delta \sigma_{k}\right)
$$

Subscript $\mathrm{k}$ represents the $\mathrm{k}_{\mathrm{th}}$ iteration in the sub-Newton iteration loop to compute trial stress. The convergence of the numerical method depends on one of the following error calculations to be lower than a prescribed tolerance.

$$
\begin{aligned}
& e_{1}=\left|\Delta \epsilon_{t}-\Delta \epsilon_{p}\left(\sigma_{t r}\right)-\Delta \epsilon_{e}\left(\sigma_{t r}\right)\right| \\
& e_{2}=\left|\frac{\Delta \epsilon_{t}-\Delta \epsilon_{p}\left(\sigma_{t r}\right)-\Delta \epsilon_{e}\left(\sigma_{t r}\right)}{\left|\Delta \epsilon_{t}\right|}\right| \\
& e_{3}=\frac{\left|\Delta \epsilon_{t, k}-\Delta \epsilon_{p, k}\left(\sigma_{t r, k}\right)-\Delta \epsilon_{e, k}\left(\sigma_{t r, k}\right)\right|}{\left|\Delta \epsilon_{t, l}-\Delta \epsilon_{p, l}\left(\sigma_{t r, l}\right)-\Delta \epsilon_{e, l}\left(\sigma_{t r, l}\right)\right|}
\end{aligned}
$$

where;

$\sigma_{t r}$ is the trial stress computed in VPSC,

$\Delta \sigma_{t}$ is the total incremental strain,

$\Delta \sigma_{e}$ is the incremental elastic strain,

$\Delta \sigma_{p}$ is the plastic strain.

The methodology outlined above allows for VPSC to be applied within the finite element fuel rod representation in the BISON fuel behavior code to calculate the cladding stress and strain response as a function of the applied temperature, stress, and fast neutron flux fields. The use of VPSC to calculate the strain rate within the integration point volumes though out the cladding replaces the empirical constitutive models outline in Section 1. The mechanistic representation of the processes controlling material deformation contained in VPSC will more directly represent the microstructure characteristics (texture) and microstructure evolution (irradiation damage) that occurs in the cladding during operation in the reactor. 


\subsection{Benchmark cases}

Four different benchmark cases were used to test the functionality of BISON/VPSC to exchange the important state and field variables and to conduct the solution algorithm in the manner that was envisioned. The results of the benchmark cases have been compared to results from the standalone version of VPSC. Table 2 below lists all the benchmark cases used for the tests. A single 3-D eight-node element with a uniform stress, temperature, and radiation field has been used for all the test cases. The finite element methodology divides the 3-D element into 8 volume regions in which VPSC is called to calculate the strain rate arising from the applied forcing functions.

Table 2: List of test cases for benchmarking BISON/VPSC

\begin{tabular}{|c|l|c|c|c|}
\hline $\begin{array}{c}\text { Case } \\
\text { Number }\end{array}$ & Description & Temperature, K & $\begin{array}{c}\text { Stress, MPa } \\
\text { (Direction) }\end{array}$ & Texture \\
\hline 1 & Thermal creep & 523 & $10(\mathrm{Y})$ & $(0,0,0)^{\dagger}$ \\
\hline 2 & Instantaneous plasticity & 523 & $100(\mathrm{Y})$ & four-grain \\
\hline 3 & Irradiation creep and growth & 523 & $1(\mathrm{Y})$ & $(0,0,0)$ \\
\hline 4 & Creep with large deformation & 1073 & $200(\mathrm{X})$ & four-grain \\
\hline
\end{tabular}

†The texture file describes the fraction of crystals with a given orientation in terms of Euler angles of three consecutive rotations: a) The first is the angle of rotation around the sample axis $\mathrm{Z}$; b) The second is the angle of rotation around the rotated crystal axis X ; c) The third is the angle around the axis $\mathrm{Z}$ in the new coordinate system.

$$
\ddagger \text { Dose rate }=1.0 \times 10^{-7} \mathrm{dpa} / \mathrm{sec}
$$

The 4-grain texture basal poles are shown in Figure 6. The orientation of the four grains is described using three Euler angles in Table 3. The same weight is assigned to each of the crystallographic directions, which is consistent with the orthotropic symmetry of the texture.

Case 1 and Case 2 exercised the thermal creep and the instantaneous plasticity capabilities under constant temperature and stress conditions. The results from these two cases showed that the finite element implementation of VPSC reproduced the results from the standalone version. Testing of the irradiation creep and growth model (Case 3 in Table 2) found that the irradiation growth strains are very similar between the two approaches. Differences were noted however, that can be attributed to the method used to calculate strain rate from irradiation growth in an engineering scale analysis versus the point model approach used in VPSC. Future work will identify an appropriate scheme to use VPSC at the engineering scale for irradiation growth. Case 4 was prepared to test the modeling of large deformation by applying high temperature and high stress conditions. The comparison of the calculated plastic strain is shown in Figure 12 for the finite element approach and standalone version of VPSC. The results show excellent agreement between the two approaches.

The results from the benchmark cases demonstrate that the Newton-Raphson iteration method used to couple the mechanism-based microstructure model to the finite element solution has been properly implemented into the multiphysics fuel code BISON. The approach used to couple VPSC and BISON uses the microstructure model to compute the trial stress for a given input of total strain received from the engineering scale mechanics calculation. The micromechanical model calculates the contribution to the total strain increment from each of the mechanistic deformation modes active, including thermal and irradiation creep, irradiation growth, and instantaneous plasticity. Furthermore, a procedure was developed to reduce

Table 3: Four-grain texture described by Euler angles

\begin{tabular}{|c|c|c|c|}
\hline & $\begin{array}{c}\text { First Euler } \\
\text { Angle (degree) }\end{array}$ & $\begin{array}{c}\text { Second Euler } \\
\text { Angle (degree) }\end{array}$ & $\begin{array}{c}\text { Third Euler } \\
\text { Angle (degree) }\end{array}$ \\
\hline Grain 1 & 138.63 & 80.30 & 0.00 \\
\hline Grain 2 & 138.63 & 99.70 & 0.00 \\
\hline Grain 3 & 221.37 & 80.30 & 0.00 \\
\hline Grain 4 & 221.37 & 99.70 & 0.00 \\
\hline
\end{tabular}




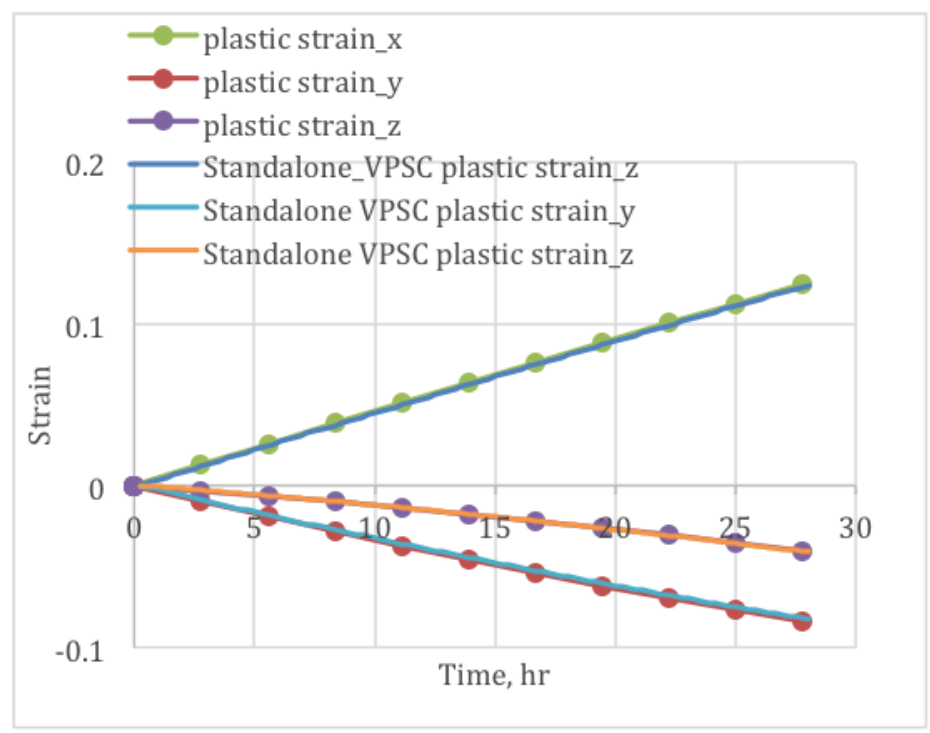

Figure 12: Comparison between BISON/VPSC and standalone VPSC for high temperature creep using a four-grain polycrystal representation

the number of grains included in the VPSC calculation that still provides a representative crystallographic texture with less computational requirements.

\section{Demonstration of Advanced Material Modeling for Cladding Thermo-Mechanical Behavior}

This section presents the results of two cases for testing the mechanism-based model using BISON/VPSC. The first case describes the results of modeling irradiation growth with emphasis on the crystallographic texture effect. The second case describes the results of modeling large deformation at high temperatures to demonstrate the codes capability of modeling clad ballooning in the postulated LOCA condition.

\subsection{Irradiation Growth}

Calculation of fuel rod elongation during irradiation is an important function of engineering scale fuel performance codes. The anisotropic properties of zirconium alloys can result in a strong dependency of irradiation growth on the crystallographic texture, which can be accounted for in the mechanism based model in VPSC. The results from an empirical model, which also accounts for crystallographic texture effects, is presented for comparison. Specifically, the empirical model used for comparison is the Franklin model, which was derived from an extensive irradiation and post-irradiation examination program performed in the 1970's to obtain data on Zircaloy-4 cladding axial elongation, circumferential creep, ovalization, and ridging $[6]$.

\subsubsection{Texture Effect in Empirical Models}

Franklin performed a regression analysis on cladding elongation measurements from 144 full-length fuel rods or empty tubes irradiated under a variety of PWR conditions. Several empirical models were developed correlating the fractional length change with the fast neutron fluence and a number of design and material parameters. The correlation that includes the texture effect can be summarized in the following equation:

$$
\frac{\Delta L}{L}=3.69 \times 10^{-17}(\phi t)^{0.655}\left[\cos \left(\theta_{\max }\right)\right]^{1.75}
$$

where;

$\Delta L$ is the rod length change $(\mathrm{m})$, 
$L$ is the rod length (m),

$\phi t$ is the fast neutron fluence $\left(n / \mathrm{cm}^{2}\right), E>1 M e V$, and

$\theta_{\max }$ is the angle between the maximum intensity of basal pole and the radial direction in the radialcircumferential plane (degree).

The effect of texture is reflected in a single parameter $\theta_{\max }$ in the empirical model. The reported $\cos \left(\theta_{\max }\right)$ of the characterized rods ranges from 0.6427 to 0.8746 . The correlation shows that the fractional length increase is proportional to $\cos \left(\theta_{\max }\right)^{1.75}$ when fast fluence and $\cos \left(\theta_{\max }\right)$ are used as independent variables.

\subsubsection{VPSC Analysis Using Two Grain Representation}

The texture effect on the irradiation growth was examined using the two-grain texture representation with basal poles on the radial-circumferential plane. By varying the orientation of each grain, different texture is created for the simulation. Table 4 shows the input crystallographic parameters for the different textures used for the analysis.

Table 4: Input crystallographic parameters for each texture used in the VPSC analysis

\begin{tabular}{|c|c|c|}
\hline$\theta_{\max }$ (degree) & $\mathrm{fr}^{a}$ & Euler angles (degree) of rotation \\
\hline 30 & 0.7500 & $(150,90,0),(210,90,0)$ \\
\hline 35 & 0.6710 & $(145,90,0),(215,90,0)$ \\
\hline 40 & 0.5868 & $(140,90,0),(220,90,0)$ \\
\hline 45 & 0.5000 & $(135,90,0),(225,90,0)$ \\
\hline 50 & 0.4132 & $(130,90,0),(230,90,0)$ \\
\hline \multicolumn{2}{|c}{ fraction of basal poles in radial direction } \\
\hline
\end{tabular}

Different initial dislocation densities $\left(\rho_{d}=1.67 \times 10^{11} / \mathrm{m}^{2}\right.$ and $\rho_{d}=1.67 \times 10^{12} / \mathrm{m}^{2}$ respectively $)$ are used to represent cladding alloys with different levels of cold work reduction from the fabrication process. At the irradiation dose of $5 \mathrm{dpa}$ (neutron dose to a fast fluence equivalence is $\sim 1 \times 10^{22} \mathrm{n} / \mathrm{cm}^{2}$ ), all irradiation growth strains are normalized by the growth strain computed at $\theta_{\max }=30^{\circ}$. A comparison between the Franklin engineering model results and the BISON-CASP/VPSC calculations is shown in the Figure 13 where the normalized growth strain is plotted as a function of $\cos \left(\theta_{\max }\right)$ for $\theta_{\max }$ values ranging between $50^{\circ}$ and $30^{\circ}$. It appears that the two approaches only have agreement at higher values of $\cos \left(\theta_{\max }\right)$, and a large discrepancy is noticeable at lower values of $\cos \left(\theta_{\max }\right)$ or larger $\theta_{\max }$.

The results shown in Figure 13 indicate that the VPSC model calculates a smaller effect of texture on the growth strain as compared to the Franklin model. Several factors could be the cause of the differences in the dependence of the growth strain on texture calculated by the two approaches. First,the use of the 2 -grain representation in VPSC produces a Kearns axial factor $\left(\mathrm{f}_{z}\right)$ that is equal to zero. Several have shown that irradiation growth is proportional to the Kearns factor in the desired direction, thus the VPSC results will by default display a weak dependence on changes in texture. Second, the amount of data available to Franklin to determine a texture dependency was limited, which could result in a large uncertainty in the coefficients used to represent texture in the model. The texture dependency of the Franklin model appears to be stronger than the classical effect given by $\frac{\Delta L}{L} \propto 1-3 f_{z}$ would suggest for the expected texture variations. Lastly, the amount of cold work varied between the cladding tubes in the irradiation growth database used by Franklin. The resulting dislocation densities would have been different in these tubes, potentially changing the irradiation behavior. The discrepancy of the results between the mechanistic-based model and the empirical model highlights the difference in the two modeling approaches, indicating more work is needed to better model the role of texture on irradiation growth. 


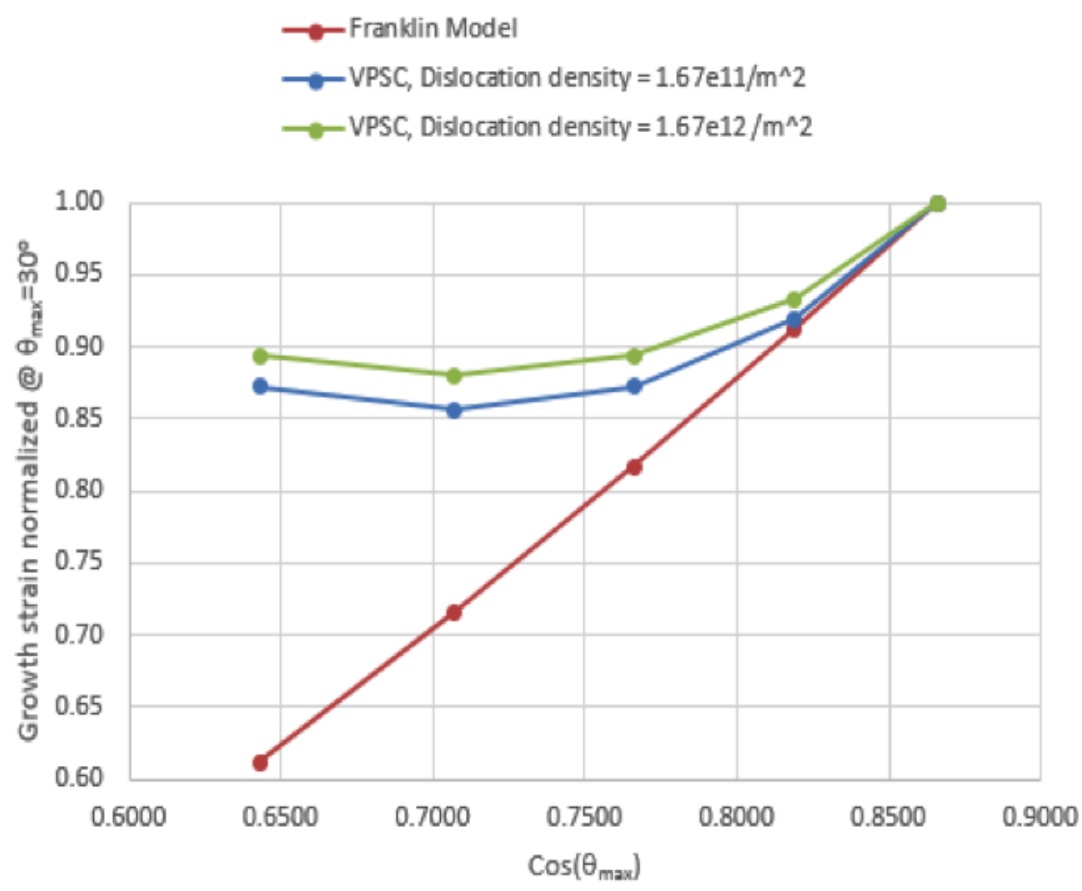

Figure 13: Comparison between the engineering model and VPSC results in modeling irradiation growth at different $\theta_{\max }$ at 5 dpa

\subsection{High Temperature Creep Test}

During a postulated LOCA, the conditions external to the fuel rod lead to significant heat up of the cladding and decrease in coolant pressure. Cladding temperatures can range from $600^{\circ} \mathrm{C}$ to $1200^{\circ} \mathrm{C}$, depending on a variety of factors. Under high internal rod pressure conditions, thermal creep and plastic deformation of the cladding can lead to large localized strain accumulation, a phenomenon called ballooning[9]. Clad ballooning is important during a LOCA as the cladding expansion will cause reduction and blockage of the coolant channel, adversely impacting the coolant flow and heat transfer conditions. Transient fuel behavior modeling is used to calculate the cladding deformation during ballooning in order to account for the impact of irradiation on rod internal pressure and fuel rod stored energy. Several empirical correlations for the thermal creep rate of zirconium alloys have been obtained from experimental data. These models have been used to estimate the burst stress and burst strain data as shown by Jahingir et al. using Falcon [50].

\subsubsection{Creep Properties at High Temperatures}

As described in Section 2.3, the current material parameters for the creep model in VPSC for zirconium alloys are benchmarked against thermal creep data at temperatures below $596 \mathrm{~K}$. At high temperatures representative of a LOCA, the microstructure material model parameters need to be re-calibrated. For the purpose of this demonstration, the approach taken to model viscoplastic deformations at high temperature is to make an ad-hoc change in the activation energy used for thermal creep (Equation 24). Creep rates calculated by the standalone-VPSC have been compared to the creep rates by other models $[9,15]$ to provide a reference for the calibration. For modeling high temperature creep behavior in VPSC, a value of $\mathrm{Q} / \mathrm{R}=18000 \mathrm{~K}$ is used instead of the $\mathrm{Q} / \mathrm{R}=10000 \mathrm{~K}$ at $\mathrm{T}=596 \mathrm{~K}$ in Equation 24 .

\subsubsection{BISON/VPSC Analysis of High Temperature Creep}

A test case to model a segment of clad tube axially constrained was prepared to evaluate the capabilities of BISON/VPSC to simulate large-strain deformation during a LOCA. The key input parameters are provided 
in Table 5 and Figure 14. The boundary and initial conditions consist of a constant pressure of $20 \mathrm{MPa}$ on the inside surface of the tube and an applied average temperature ramp of $0.8 \mathrm{~K} / \mathrm{sec}$ starting at an initial temperature of $810 \mathrm{~K}$. The mechanical boundary conditions at the top and bottom of the tube only allow for radial displacements, with no axial expansion or contraction occurring during the simulation. These mechanical constraints result in a biaxial stress state within the tube with an axial stress that is much larger than a closed tube condition $\left(\sigma_{\text {axial }}=\frac{1}{2} \sigma_{\text {hoop }}\right)$. A peak-to-average temperature ratio of 1.012 is applied at the tube mid-height. This temperature profile produces a $30^{\circ} \mathrm{C}$ temperature variation in the axial direction

Table 5: Input parameters for the high temperature creep test case

\begin{tabular}{|l|c|c|}
\hline Parameter & Unit & Value \\
\hline Number of elements & - & 5 \\
\hline Element type & - & QUAD4 axisymmetric \\
\hline Internal pressure & $\mathrm{MPa}$ & 20 \\
\hline External pressure & $\mathrm{MPa}$ & 0 \\
\hline Texture & - & Four-grain \\
\hline Time dependent average temperature & $\mathrm{K}$ & $810-1073$ \\
\hline Simulation time & $\mathrm{Hr}$ & 0.12 \\
\hline Peak (temperature) to average ratio & - & 1.012 \\
\hline Q/R & $\mathrm{K}$ & 18,000 \\
\hline
\end{tabular}
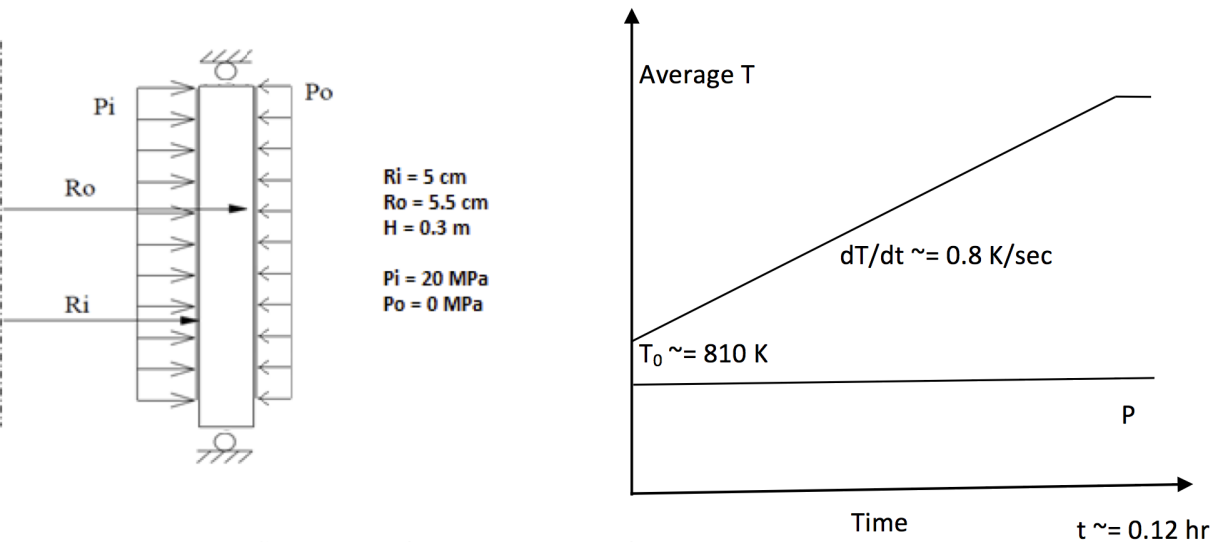

Figure 14: Schematic of input conditions for testing high temperature creep

Results of the total hoop, radial, and axial strain computed using BISON/VPSC are shown in Figure 15. The results indicate a significant amount of wall thinning as represented by the equal amount of strain in the hoop and radial directions $\left(\epsilon_{\text {hoop }} \simeq \epsilon_{\text {radial }}\right)$. The wall thinning is further observed in Figure 16 which shows the deformed geometry at the end of simulation under the imposed axial temperature profile. Figure 17 compares the hoop strain between the mid-height element with the largest deformation and the top element with relatively smaller deformation.

These results demonstrate that large deformation due to accumulated creep strains can be simulated throughout the whole segment of the cladding tube under rod internal pressure using a mechanistic microstructure evoluation model. Initially, the deformation at the mid-height element with higher temperature and the top/bottom element with lower temperature are similar; the major difference is due to different creep rates caused by the temperature variation. When the deformation becomes large (hoop strain $>5 \%$ ), both the temperature and the deformed geometry contribute to the deformation rate, leading to an acceleration of the strain accumulation. This result is consistent with experimental data obtained by Erbacher, et al. who 
demonstrated that azimuthal temperature variations of $\sim 25 \mathrm{~K}$ are sufficient to reduce the circumferential average ballooning strain by a factor of two [9].

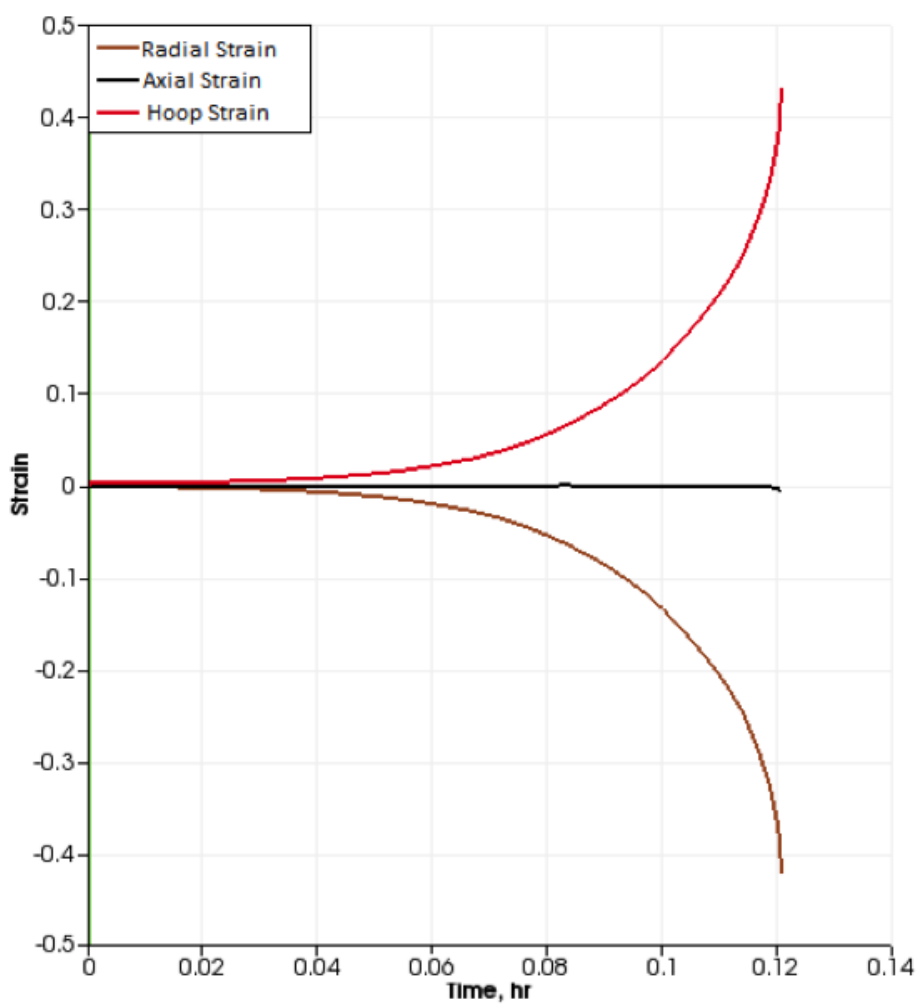

Figure 15: Total strains versus time at the mid-height element

\subsection{Observations}

These results highlight the advantages of an advanced polycrystal constitutive law for modeling irradiation growth and large-strain thermal creep deformation using a mechanistic microstructure model within BISON. Comparison of the empirical models with the results from the reduced texture VPSC model using two grains has highlighted the differences between the microstructure model and the empirical correlation. The results suggest that the mechanism-based microstructure model may provide more information about the basic processes leading to deformation than the empirical modeling approach. The results from the high temperature thermal creep analysis of large-strain clad deformation in a postulated LOCA condition has shown that under an imposed axial temperature profile, the localized clad thinning (or ballooning) can be simulated using BISON/VPSC.

\section{Summary and Future Work}

The results of these investigations have demonstrated the potential of incorporating a microstructurallybased deformation model for zirconium alloy materials under conditions of stress, temperature, and irradiation to improve thermo-mechanical simulations of LWR fuel rods under steady state and transient conditions. By coupling the VPSC material model into the BISON engineering scale fuel performance code, the role of microstructural effects, such as crystallographic texture, vacancy and interstitial defects, dislocation networks, and multi-grain interactions can be accounted for when calculating the deformation behavior 

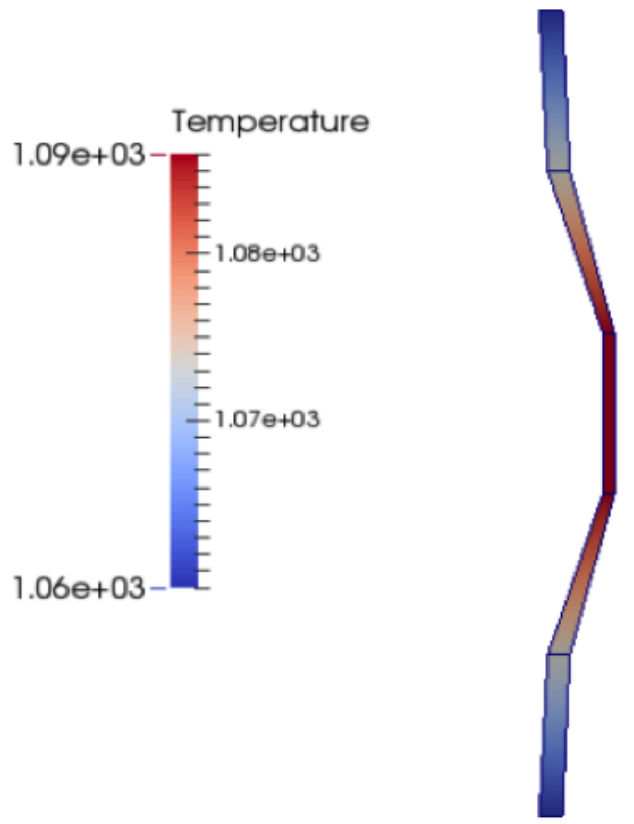

Figure 16: Temperature contour and deformed geometry at the end of simulation (Aspect ratio scaled 20x)

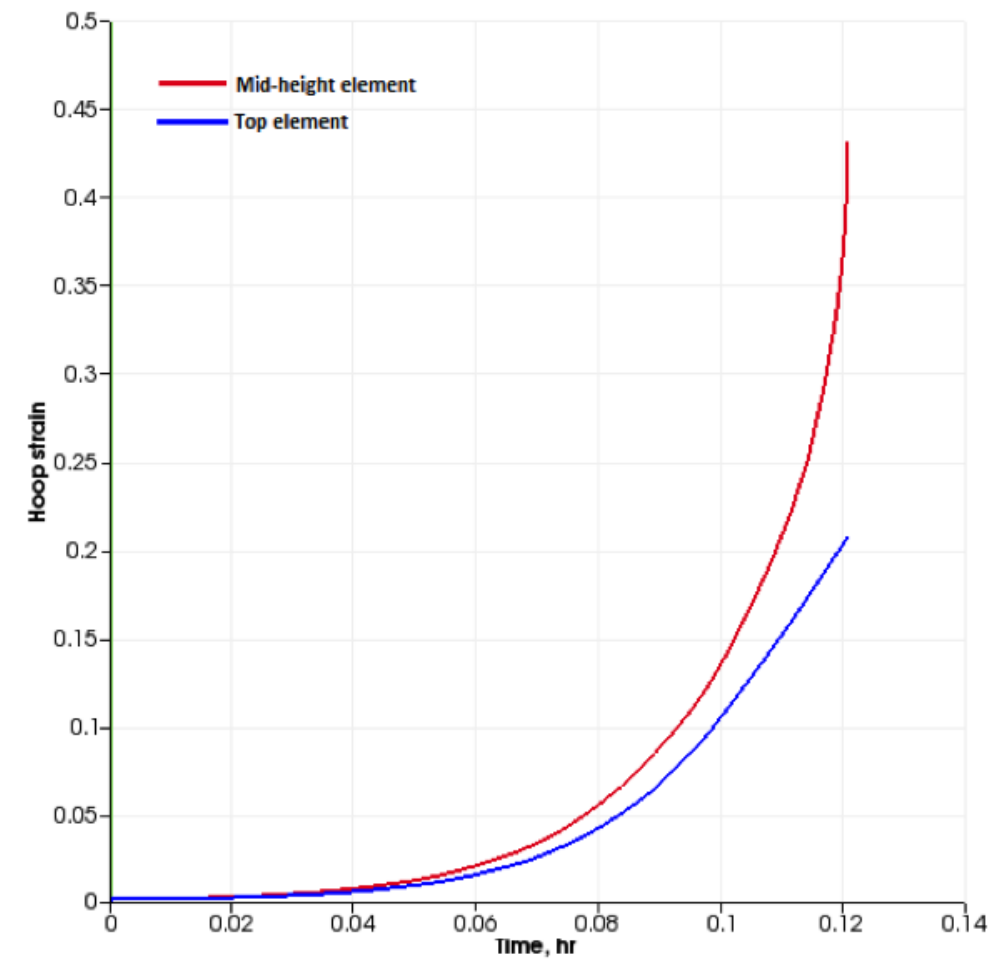

Figure 17: Comparison of hoop strain between the mid-height element and the top element

of cladding tubes during irradiation. Traditional fuel behavior modeling capabilities for cladding deforma- 
tion are built on a suite of empirical or semi-empirical material property or behavior models derived from a variety of testing and characterization programs that contain limited sets of environmental conditions or material characteristics. The computational framework resulting from this work provides a foundation to develop an improved mechanistic understanding of the microstructural characteristics that control cladding deformation. One step in that direction is the use of atomistic simulations to improve modeling of the diffusion and capture of point defects and defect clusters. Such advanced material constitutive models can allow for extension of fuel performance modeling to applications of improved cladding materials or new operating regimes where validation data is limited.

The next phase of the development activities will focus on three main areas: 1) improving the microstructural modeling, 2) numerical efficiency and functionality, and 3) expanding the material property benchmark database. Several simplifying assumptions have been employed in defining the crystallographic mechanisms to derive the constitutive equations for irradiation growth, irradiation creep, thermal creep and instantaneous plasticity. The focus will be to utilize atomistic simulations to improve representation of such mechanisms as loop nucleation as a function of alloying elements for irradiation growth and the activation energy for thermal creep at high temperatures, where complex interactions occur between dislocations and irradiation-induced loop defects. Improvements in numerical efficiency may be realized through several different approaches, including an improved pre-conditioning of the matrix solution, refinements on updating the constitutive model conditions, and enabling parallel processing functionality. Lastly, expanding the comparison of the capability to mechanical data obtained from irradiation cladding is necessary to benchmark the polycrystal model for coupled irradiation growth and creep behavior.

\section{Acknowledgments}

This work was supported by the Consortium for Advanced Simulation of Light Water Reactors (www.casl.gov), an Energy Innovation Hub (http://www.energy.gov/hubs) for Modeling and Simulation of Nuclear Reactors under U.S. Department of Energy Contract No. DE-AC05-00OR22725.

\section{References}

[1] Lebensohn, R. and Tomé, C.N., Acta Metallurgica et Materialia 41, 2611-2624. (1993).

[2] Montgomery, et. al., "Peregrine: Advanced Modeling of Pellet-Cladding Interaction (PCI) Failure in LWRs," Proceedings of the TopFuel 2012 Reactor Fuel Performance Meeting, Manchester, U.K., Sep. 2-6, (2012).

[3] M. Billone, R. Montgomery, Y.R. Rashid, and J. Head, "Advancements in the behavioral modeling of fuel elements and related structures," Nucl. Engrg. Des. 134, (1992), 23-36.

[4] K. L. Murty, I. Charit, Texture development and anisotropic deformation of zircaloys, Progress in Nuclear Energy, Volume 48, Issue 4, May 2006, Pages 325-359.

[5] R.A. Holt, "In-reactor deformation of Zirconium alloy components", J of ASTM Int, Vol 5 No. 6 (2008)

[6] D. G. Franklin, "Zircaloy-4 Cladding Deformation during Power Reactor Irradiation," ASTM STP 754, American Society for Testing and Materials, 1982, pp.235-267.

[7] V. Fidleris, "The irradiation creep and growth phenomena", J Nuclear Mats 159 (1988) 22.

[8] Y. Matsuo, "Thermal Creep of Zircaloy-4 Cladding under Internal Pressure," Journal of Nuclear Science and Technology, 24:2, p111-119, 1987

[9] F. J. Erbacher, H.J. Neitzel, H. Rosinger, H. Schmidt, and K. Wiehr, "Burst Criterion of Zircaloy Fuel Claddings in a Loss-of-Coolant Accident," ASTM 754, American Society for Testing and Materials, 1982, pp. 271-283.

[10] R. O. Montgomery, W. F. Lyon, M. N. Jahingir, and S. Yagnik, "Capabilities of the FALCON Steady State and Transient Fuel Performance Code", Proceedings of the 2004 International Meeting on LWR Fuel Performance, Orlando FL, September 19-22, 2004.

[11] N. E. Hoppe, "Engineering Model for Zircaloy Creep and Growth," Proceedings of the ANS-ENS International Topical Meeting on LWR Fuel Performance, p. 201-209, Avignon, France, April 21-24 (1991).

[12] K. Lassmann, "The structure of fuel element codes," Nucl. Engrg. Des. 57 (1980) 17-39.

[13] K.L. Murty, et al., "Thermal Creep of Zircaloy-4 Cladding," Presented to 4th Int. Conf. on Structural Mechanics in Reactor Technology, San Francisco, Aug. 15-19, 1977.

[14] D.G. Franklin, G.E. Lucas, A.L. Bement, Creep of Zirconium Alloys in Nuclear Reactors, STP 815, ASTM, Philadelphia, PA, 1983.

[15] M. Limback, T. Andersson, "A Model for Analysis of the Effect of Final Annealing on the In- and Out-of-Reactor Creep Behavior of Zircaloy Cladding," Zirconium in the Nuclear Industry: Eleventh International Symposium, ASTM STP 1295, American Society for Testing and Materials, 1996, pp. 448-468. 
[16] K.E. Amin, "A Universal Law for High-Temperature Diffusion Controlled Transient Creep," J. Mech. Phys. Solids, Vol 18, pg 413-426, 1970.

[17] D. T. Hagrman, "MATPRO - A Library of Materials Properties for Light Water Reactor Accident Analysis," SCDAP/RELAP5/MOD3.1 Code Manual, Vol 4, NUREG/CR-6150, EGG-2720, June (1995).

[18] R.A. Holt, R.W. Gilbert, " $<\mathrm{c}>$-Component dislocations in annealed Zircaloy irradiated at about 570 K," Journal of Nuclear Materials, Volume 137 (1986) pp 185-189.

[19] M. Griffiths, R.A. Holt, A. Rogerson, Microstructural aspects of accelerated deformation of Zircaloy nuclear reactor components during service, Journal of Nuclear Materials, Volume 225 (1995) 245-258.

[20] R.A. Holt, "Mechanisms of Irradiation Growth of Alpha-Zirconium Alloys," Journal of Nuclear Materials, Volume 159 (1988) pp 310-338.

[21] C.H. Woo, J of Nuclear Materials 131 (1985) 105.

[22] K. Geelhoood, et. al., "FRAPCON-3.4: A Computer Code for the Calculation of Steady-State Thermal-Mechanical Behavior of Oxide Fuel Rods for High Burnup," NUREG/CR-7022, Vol. 1, 2011.

[23] S. Yip, Handbook of Materials Modeling, Dordrecht: Springer, 2005, ISBN 978-1-4020-3287-5.

[24] R. Montgomery, C. Stanek, W. Liu, and B. Kendrick, "US DOE CASL Program Fuel Performance Modeling for Steady State and Transient Analysis of LWR Fuel," Proceedings of the IAEA Technical meeting on modelling of water-cooled fuel including design basis and severe accidents, October 28 - November 1, 2013, Chengdu, China.

[25] D. Gaston, C. Newman, G. Hansen, and D. Lebrun-Grandié. MOOSE: A parallel computational framework for coupled systems of nonlinear equations. Nucl. Eng. Design, 239, p. 1768-1778, 2009.

[26] R. Williamson, J. Hales, S. Novascone, M. Tonks, D. Gaston, C. Permann, D. Andrs, and R. Martineau, "Multidimensional multiphysics simulation of nuclear fuel behavior," Journal of Nuclear Materials 423(2012) 149-163.

[27] I.J. Beyerlein and C.N. Tomé, "A dislocation based constitutive law for pure Zr including temperature effects", Int J of Plasticity 24 (2008) 867-895

[28] G. Subramanian, D. Perez, B.P. Uberuaga, C.N. Tomé, A.F. Voter, "Method to account for arbitrary strains in kinetic Monte Carlo simulations", Physical Rev B87 (2013) 144107.

[29] R.A. Lebensohn, Y. Liu, P. Ponte Castaneda, On the accuracy of the self-consistent approximation for polycrystals: comparison with full-field numerical simulations, Acta Materialia, Volume 52, Issue 18, 5347-5361 (2004).

[30] R. A. Holt, "In-reactor deformation of cold-worked Zr-2.5Nb pressure tubes," Journal of Nuclear Materials 372 (2008) $182-214$.

[31] A. Rogerson, "Irradiation growth in zirconium and its alloys", Journal of Nuclear Materials 159 (1988) 43-61.

[32] J.J Kearns, On the relationship among ' $\mathrm{f}$ ' texture factors for the principal planes of zirconium, hafnium and titanium alloys, Journal of Nuclear Materials, Volume 299, Issue 2, November 2001, Pages 171-174.

[33] Subramanian, G. and Tomé, C.N., Progress report on the incorporation of lower length scales into polycrystal plasticity model, Report LA UR 12-25612 (2012).

[34] S.I. Golubov, A.V. Barashev, R.E. Stoller, "On the origin of radiation growth in HCP crystals", ORNL/TM-2011/473 (2011).

[35] S.I. Golubov, A.V. Barashev, R.E. Stoller, B.N. Singh, "Breakthrough in understanding radiation growth in Zirconium", ASTM STP (2013), in press.

[36] A.V. Barashev, S.I. Golubov, and R.E. Stoller, Theoretical investigation of microstructure evolution and deformation of zirconium under cascade damage conditions, Technical Report ORNL/TM-2012/225 Oak Ridge National Laboratory (2012)

[37] A. Rogerson and R.H. Zee, "High fluence irradiation growth in single crystal zirconium at 553K", J. Nuclear Materials 151 (1987) 81-83 P.M. Sargent, M.F. Ashby, "Deformation maps for titanium and zirconium", Scripta Metall 16 (1982) $1415-22$.

[38] J.H. Gittus, "Theory of dislocation-creep due to the Frenkel defects or interstitialcies produced by bombardment with energetic particles", Phil Mag 25 (1972) 345-354.

[39] Heald PT and Harbottle JP, Journal of Nuclear Materials 67, p. 229 (1977).

[40] N. Christodoulou, P.A. Turner, C.N. Tomé, C.K. Chow, R.J. Klassen, "Analysis of steady-state thermal creep of Zr-2.5\% Nb pressure tube material", Metall. and Material Trans. 33A (2002) 1103-15.

[41] C. Deo, C. N. Tomé, R. Lebensohn, S. Maloy, (2008) J of Nuclear Materials 377, 136-140

[42] Le Saux, M. L., Besson, J., Carassou, S., Poussard, C., Averty, X. (2008) J of Nuclear Materials 378, 60-69.

[43] G. Lucas and R. Pelloux, (1981) Metallurgical Transactions A12, 1321-31.

[44] T. Onchi, H. Kayano, Y. Higashiguchi, (1977) J of Nuclear Science and Technology 14, 359-369

[45] B. Cazalis, J. Desquines (2007) Nuclear Technology 157, 215-229

[46] G.I. Taylor: Proc. R. Soc. London A, 1934, vol. A145, p. 362.

[47] A. Arsenlis, B.D. Wirth, M. Rhee, (2004) Philosophical Magazine 84, 3617-3635

[48] A. Arsenlis, M. Rhee, G. Hommes, R. Cook, and J. Marian, (2012) Acta Materialia 60, 3748-3757

[49] J. Segurado, R. A. Lebensohn, J. LLorca, and C. N. Tomé, "Multiscale modeling of plasticity based on embedding the viscoplastic self-consistent formulation in implicit finite elements," International Journal of Plasticity 28 (2012) 124-140.

[50] M. N. Jahingir, J. Alvis, R. O. Montgomery, and O. Ozer, "Analysis of Fuel Behavior During LOCA Tests Using FALCON MOD01," Proceedings of the 2005 Water Reactor Fuel Performance Meeting, Kyoto, Japan, October 2-6, 2005. 